\title{
Partial regularity for weak solutions of nonlinear elliptic systems: the subquadratic case
}

\author{
Lisa Beck*
}

\begin{abstract}
We consider weak solutions of second order nonlinear elliptic systems of divergence type under subquadratic growth conditions. Via the method of $\mathcal{A}$-harmonic approximation we give a characterization of regular points up to the boundary which extends known results from the quadratic and superquadratic case. The proof yields directly the optimal higher regularity on the regular set.
\end{abstract}

\section{Introduction}

In this paper we study weak solutions $u \in W^{1, p}\left(\Omega, \mathbb{R}^{N}\right), p \in(1,2)$, of nonlinear homogeneous elliptic systems of second order in divergence form

$$
\operatorname{div} A(\cdot, u, D u)=0 \quad \text { in } \Omega,
$$

where $\Omega \subset \mathbb{R}^{n}, n \geq 2$, is a bounded domain, and where the coefficients $A: \bar{\Omega} \times \mathbb{R}^{N} \times \mathbb{R}^{n N} \rightarrow \mathbb{R}^{n N}$ satisfy reasonable structure conditions of so-called subquadratic growth stated in the next section.

We first give a short overview of partial regularity results in the interior and at the boundary. For detailed discussions we refer to [15], [19] and [24], where examples and motivations can be found explaining the development of regularity theory and the idea of partial regularity throughout the last century.

In 1968, De Giorgi demonstrated in [9] that, in contrast to equations, we cannot in general expect a weak solution to a nonlinear system to be a classical one (i. e., of class $C^{2}$ ). The best we can hope for is partial regularity, in other words that there exists a set $\Omega_{0} \subset \Omega$ such that $\Omega \backslash \Omega_{0}$ is of Lebesgue measure zero and that $u$ or even $D u$ is locally regular (e.g. Hölder-continuous) in $\Omega_{0}$. There are different approaches to prove partial regularity in the interior: Giaquinta-Modica [16] and Ivert [23] were the first to utilize the direct method; the blow-up technique was earlier applied in the setting of elliptic systems by Giusti-Miranda [18]; furthermore, Duzaar and Grotowski introduced in [10] the method of $\mathcal{A}$-harmonic approximation, which is based on Simon's proof of the regularity theorem of Allard.

In the situation considered in this paper we will use a version of the latter technique which has been applied to various situations concerning regularity in the past few years. Here it is adapted to the subquadratic case and to the boundary setting simultaneously. First we show that a function $g$ which is approximately $\mathcal{A}$-harmonic, i. e., for which $f_{B_{R}\left(x_{0}\right)} \mathcal{A} D g \cdot D \varphi d x$ is sufficiently small for all test functions $\varphi \in C_{0}^{1}\left(B_{R}\left(x_{0}\right), \mathbb{R}^{N}\right)$, is close to an $\mathcal{A}$-harmonic function $h$ in the $L^{p}$-sense. In this context we do not prescribe boundary values for $h$. These values are thus taken in a natural way. In the next step we consider the frozen system with coefficients $\mathcal{A}=D_{z} A\left(x_{0},(u)_{x_{0}, R},(D u)_{x_{0}, R}\right)$. We compare an approximately $\mathcal{A}$ harmonic rescaling of the solution $u$ to the original system with an $L^{p}$-close $\mathcal{A}$-harmonic function $h$. Using good a priori estimates for $h$ and a Caccioppoli-type inequality, we then find an excess-decayestimate in points where certain smallness assumptions (see below) are satisfied. Finally, by Campanato's characterization of Hölder-continuous functions, we conclude the desired partial regularity criterion.

Apart from the $\mathcal{A}$-harmonic approximation lemma all proofs are direct. This gives a good control on the dependencies on the structure conditions and enables us to obtain the optimal regularity result in a direct

${ }^{*}$ L. Beck, Mathematisches Institut der Friedrich-Alexander-Universität Erlangen-Nürnberg, Bismarckstr. 1 1/2, 91054 Erlangen, Germany. E-mail: beck@mi.uni-erlangen.de 
way. The result is optimal in the following sense: if $\left(1+|z|^{2}\right)^{\frac{1-p}{2}} A(x, u, z)$ is uniformly Hölder-continuous in $x$ and $u$ with exponent $\alpha$, then $D u$ is partially Hölder-continuous with the same exponent $\alpha$. The following example demonstrates that we cannot expect higher regularity than the one allowed by the regularity of the coefficients (see [20], Example 1.1 in the case $p=2$ ):

Example: Let $n \geq 2, N=1$ and $\alpha \in(0,1)$. Define coefficients $A(x, z)$ for $x, z \in \mathbb{R}^{n}$ by

$$
A(x, z)=\frac{\left(1+|z|^{2}\right)^{\frac{p-2}{2}} z}{\left(1+\left(1+x_{n}^{\alpha}\right)^{2}\right)^{\frac{p-2}{2}}\left(1+x_{n}^{\alpha}\right)} .
$$

Then the coefficients fulfill (H1) - (H4) of chapter 2 for the domain $\Omega$ given by an appropriate smoothing of $B^{+}$. The function $u(x)=\frac{1}{1+\alpha} x_{n}^{1+\alpha}+x_{n}$ is a weak solution of $\operatorname{div} A(x, D u)=0$ on $\Omega$ of $\operatorname{class} C^{1, \alpha}(\Omega, \mathbb{R})$, but not more regular.

In the subquadratic case, where $u \in W^{1, p}\left(\Omega, \mathbb{R}^{N}\right)$ with $p \in(1,2)$ and where the coefficients $A$ satisfy a corresponding $(p-1)$-growth condition, only few partial regularity results are known. In [25] the blow-up technique is applied to a special quasi-linear system to show Hölder-continuity in the interior situation. The quasi-linearity further allows us to infer that the singular set is of $(n-p)$ Hausdorff measure zero. For minimizers of quasiconvex integrals partial regularity is studied in [5] and [6]. In [27] solutions of nonlinear systems are considered, but the regularity result is not optimal in the above sense. Theorem 2.1 closes this gap since we show the analogous (optimal) result to that of the quadratic case (see e.g. [18] combined with [21]) and to that of the superquadratic case $p \geq 2$ (see [22]). Furthermore, we give a similar characterization of regular points where the set of regular points is defined by

$$
\operatorname{Reg} u:=\left\{x \in \Omega: D u \in C^{0}\left(U, \mathbb{R}^{N}\right) \text { for some neighbourhood } U \text { of } x\right\} \text {. }
$$

More precisely we obtain that (under structure conditions introduced in chapter 2) $u \in C_{l o c}^{1, \alpha}\left(\operatorname{Reg} u, \mathbb{R}^{N}\right)$, and the set of singular points Sing $u:=\Omega \backslash \operatorname{Reg} u \subset \Pi_{1} \cup \Pi_{2}$ is of Lebesgue measure zero, with

$$
\begin{aligned}
& \Pi_{1}=\left\{x_{0} \in \Omega: \liminf _{\rho \rightarrow 0^{+}} f_{B_{\rho}\left(x_{0}\right)}\left|V(D u)-(V(D u))_{x_{0}, \rho}\right|^{2} d x>0\right\}, \\
& \Pi_{2}=\left\{x_{0} \in \Omega: \limsup _{\rho \rightarrow 0^{+}}\left(\left|(u)_{x_{0}, \rho}\right|+\left|(V(D u))_{x_{0}, \rho}\right|\right)=\infty\right\}
\end{aligned}
$$

where $V(\xi)=\left(1+|\xi|^{2}\right)^{\frac{p-2}{4}} \xi$ for $\xi \in \mathbb{R}^{k}$.

The boundary situation in the case of quasi-linear systems was considered in the early 70's in [7], in which the author showed Hölder continuity of weak solutions. Further, there are some papers, in particular by Campanato, [3], and recently by Arkhipova, [1], in which the authors obtain global regularity in low dimensions. Regularity up to the boundary in the case of general systems was lately studied for the first time in [19] via the $\mathcal{A}$-harmonic approximation in the case $p=2$ and in [22] using a version of the blow-up technique for the superquadratic case. We proceed analogously to [19] and provide a similar characterization of regular boundary points defined by

$$
\operatorname{Reg}_{\partial \Omega} u:=\left\{x \in \partial \Omega: D u \in C^{0}\left(U \cap \bar{\Omega}, \mathbb{R}^{N}\right) \text { for a neighbourhood } U \text { of } x\right\} .
$$

Here we assume the boundary $\partial \Omega$ to be of class $C^{1, \tau}$ and further $u=g$ on $\partial \Omega$ for a function $g \in$ $C^{1, \beta}\left(\bar{\Omega}, \mathbb{R}^{N}\right)$.

To this end we first have to provide a priori estimates up to the boundary for weak solutions $u \in W^{1, p}$ of homogeneous linear systems with constant coefficients. Here, in the subquadratic case, the estimates on higher derivatives we require for the Sobolev embedding theorem are not immediately available. However, by smoothing combined with the $L^{p}$-theory, the subquadratic case can be handled by similar methods as in the quadratic case, and we obtain that weak solutions are smooth up to the boundary. This allows us to derive an excess-decay estimate at the boundary. Combined with the excess-decay estimate in the interior, we show that $D u$ is locally Hölder-continuous with exponent $\min \{\alpha, \beta, \tau\}$ in points $y \in \operatorname{Reg}_{\partial \Omega} u$, 
and that the set of singular boundary points $\operatorname{Sing}_{\partial \Omega} u:=\partial \Omega \backslash \operatorname{Reg}_{\partial \Omega} u \subset \widetilde{\Sigma}_{1} \cup \widetilde{\Sigma}_{2}$, where

$$
\begin{aligned}
& \widetilde{\Sigma}_{1}=\left\{y \in \partial \Omega: \liminf _{\rho \rightarrow 0^{+}} f_{\Omega \cap B_{\rho}(y)}\left|V\left(D_{\nu_{\partial \Omega}(y)}(u-g)\right)-\left(V\left(D_{\nu_{\partial \Omega}(y)}(u-g)\right)\right)_{y, \rho}\right|^{2} d x>0\right\}, \\
& \widetilde{\Sigma}_{2}=\left\{y \in \partial \Omega: \limsup _{\rho \rightarrow 0^{+}} \mid\left(V\left(D_{\nu_{\partial \Omega}(y)}(u-g)\right)_{y, \rho} \mid=\infty\right\} ;\right.
\end{aligned}
$$

here $\nu_{\partial \Omega}(y)$ denotes the inward-pointing unit normal to $\partial \Omega$ at $y$. This means that for the regularity criterion at the boundary, only the normal derivative is of importance. This enables us to observe that the regularity criteria given in [19] and in [22] are in fact equivalent; we discuss this in more detail in section 7 . We further note here that on the boundary we do not need to assume that the mean values of $u$ are bounded due to the fact that $u$ is fixed on $\partial \Omega$ via the boundary data $g$. Note that since the boundary $\partial \Omega$ itself is of Lebesgue measure zero, this does not yield the existence of regular boundary points. For this we refer to [12].

In what follows we set the main focus on the treatment of the boundary situation. We will thus only state the intermediate estimates in the interior and refer to [2] for detailed proofs and calculations, since the interior case is technically easier to handle. We concentrate on the case of homogeneous systems. However, our method also applies to the inhomogeneous case

$$
-\operatorname{div} A(\cdot, u, D u)=B(\cdot, u, D u) \quad \text { in } \Omega,
$$

where $B(\cdot, \cdot, \cdot)$ obeys either a controllable or a natural growth condition (in the latter case, we have to consider bounded solutions satisfying the assumption $\|u\|_{L^{\infty}\left(\Omega, \mathbb{R}^{N}\right)} \leq M_{u}$ such that $\left.2 L_{1}\left(M_{u}\right) M_{u}<\nu\right)$. In the last section we sketch briefly the required modifications and state the results for the inhomogeneous situation, which are completely analogous to the corresponding results in [10], [20] and [22].

\section{Notation, structure conditions and statement of the results}

We start with some remarks on the notation used below: we write $B_{\rho}\left(x_{0}\right)=\left\{x \in \mathbb{R}^{n}:\left|x-x_{0}\right|<\rho\right\}$ and $B_{\rho}^{+}\left(x_{0}\right)=\left\{x \in \mathbb{R}^{n}: x_{n}>0,\left|x-x_{0}\right|<\rho\right\}$ for a ball or an upper half-ball, respectively, centered on a point $x_{0}\left(\in \mathbb{R}^{n-1} \times\{0\}\right.$ in the latter case) with radius $\rho>0$. Sometimes it will be convenient to treat the $n$-th component of $x \in \mathbb{R}^{n}$ separately; therefore, we set $x=\left(x^{\prime}, x_{n}\right)$ where $x^{\prime}=\left(x_{1}, \ldots, x_{n-1}\right)$. Furthermore, we write

$$
\Gamma_{\rho}\left(x_{0}\right)=\left\{x \in \mathbb{R}^{n}:\left|x-x_{0}\right|<\rho, x_{n}=0\right\},
$$

for $x_{0} \in \mathbb{R}^{n-1} \times\{0\}$. In the case $x_{0}=0$ we set $B_{\rho}:=B_{\rho}(0), B:=B_{1}$ as well as $B_{\rho}^{+}:=B_{\rho}^{+}(0), B^{+}:=B_{1}^{+}$ with $\Gamma=\Gamma_{1}(0)$. We also introduce the following notation for $W^{1, p}$-functions defined on some half-ball $B_{\rho}^{+}\left(x_{0}\right)$ and which vanish (in the sense of traces) on the flat part of the boundary:

$$
W_{\Gamma}^{1, p}\left(B_{\rho}^{+}\left(x_{0}\right), \mathbb{R}^{N}\right):=\left\{u \in W^{1, p}\left(B_{\rho}^{+}\left(x_{0}\right), \mathbb{R}^{N}\right): u=0 \text { on } \Gamma_{\rho}\left(x_{0}\right)\right\} .
$$

Let $\mathcal{L}^{n}$ and $\mathcal{H}^{k}$ denote the $n$-dimensional Lebesgue measure and the $k$-dimensional Hausdorff measure, respectively. For any bounded, measurable set $X \subset \mathbb{R}^{n}$ with $\mathcal{L}^{n}(X)=:|X|>0$, we denote the mean value of a function $h \in L^{1}\left(X, \mathbb{R}^{N}\right)$ by $(h)_{X}=f_{X} h d x$, and, in particular, we use the abbreviation $(h)_{x_{0}, \rho},(h)_{x_{0}, \rho}^{+}$for the mean value on $B_{\rho}\left(x_{0}\right)$ and on $B_{\rho}^{+}\left(x_{0}\right)$, respectively. The constants $c$ appearing in the different estimates will all be chosen greater than or equal to 1 , and they may vary from line to line.

We consider a bounded domain $\Omega$ in $\mathbb{R}^{n}$, for some $n \geq 2$. The boundary of $\Omega$ is assumed to be of class $C^{1, \tau}$ for some $\tau \in(0,1)$, i.e., for any point $x_{0} \in \partial \Omega$ there exist a radius $r>0$ and a function $h: \mathbb{R}^{n-1} \rightarrow \mathbb{R}$ of class $C^{1, \tau}$ such that (up to an isometry) $\Omega$ is locally represented by $\Omega \cap B_{r}\left(x_{0}\right)=\left\{x \in B_{r}\left(x_{0}\right)\right.$ : $\left.x_{n}>h\left(x^{\prime}\right)\right\}$. Thus we can locally straighten the boundary $\partial \Omega$ via a $C^{1, \tau}$-transformation $\mathcal{T}$ defined by $\mathcal{T}\left(x^{\prime}, x_{n}\right)=\left(x_{1}, \ldots, x_{n-1}, x_{n}-h\left(x^{\prime}\right)\right)$.

In the paper we consider a general homogeneous system of second order elliptic equations in divergence form

$$
\operatorname{div} A(\cdot, u, D u)=0 \quad \text { in } \Omega .
$$


In components, this is a system of $N$ equations of the form $\sum_{\kappa=1}^{n} D_{\kappa} A_{\kappa}^{i}(\cdot, u, D u)=0$ on $\Omega$ for $i=$ $1, \ldots, N$. Unless otherwise noted, we will always assume $p \in(1,2)$ fixed.

We impose on the coefficients $A: \bar{\Omega} \times \mathbb{R}^{N} \times \mathbb{R}^{n N} \rightarrow \mathbb{R}^{n N}$ of the system (1) standard boundedness, differentiability, growth and ellipticity conditions: the functions $(x, u, z) \mapsto A(x, u, z)$ and $(x, u, z) \mapsto$ $D_{z} A(x, u, z)$ are continuous, and for fixed $L \geq 1, \nu>0$ and all triples $(x, u, z),(\bar{x}, \bar{u}, z) \in \bar{\Omega} \times \mathbb{R}^{N} \times \mathbb{R}^{n N}$ there holds that:

(H1) A has polynomial growth:

$$
|A(x, u, z)| \leq L\left(1+|z|^{p-1}\right),
$$

(H2) $A$ is differentiable with respect to $z$ with bounded and continuous derivatives:

$$
\left|D_{z} A(x, u, z)\right| \leq L
$$

(H3) $A$ is uniformly strongly elliptic:

$$
D_{z} A(x, u, z) \lambda \cdot \lambda \geq \nu\left(1+|z|^{2}\right)^{\frac{p-2}{2}}|\lambda|^{2} \quad \forall \lambda \in \mathbb{R}^{n N},
$$

(H4) There exists a modulus of continuity $\omega$ with $\omega(t) \leq \min \left(1, t^{\alpha}\right)$ and $K:[0, \infty) \rightarrow[1, \infty)$ monotone nondecreasing such that

$$
|A(x, u, z)-A(\bar{x}, \bar{u}, z)| \leq L K(|u|)\left(1+|z|^{2}\right)^{\frac{p-1}{2}} \omega(|x-\bar{x}|+|u-\bar{u}|) .
$$

Finally, we assume the following boundary condition:

(H5) $\quad g$ is in $C^{1, \beta}\left(\bar{\Omega}, \mathbb{R}^{N}\right)$.

Since the gradient $D_{z} A(x, u, z)$ is continuous, we may conclude the existence of a modulus of continuity on compact sets of $\bar{\Omega} \times \mathbb{R}^{N} \times \mathbb{R}^{n N}$, i. e., there exists a function $\chi:[0, \infty) \times[0, \infty) \rightarrow[0, \infty)$ satisfying

$$
\begin{array}{ll}
\chi(t, 0)=0 & \text { for all } t \geq 0 \\
t \mapsto \chi(t, s) & \text { is monotone nondecreasing for fixed } s \\
s \mapsto \chi^{2}(t, s) & \text { is concave and monotone nondecreasing for fixed } t
\end{array}
$$

such that for all $(x, u, z),(\bar{x}, \bar{u}, \bar{z}) \in \bar{\Omega} \times \mathbb{R}^{N} \times \mathbb{R}^{n N}$ with $|u|+|z|+|u-\bar{u}|+|z-\bar{z}| \leq M+1$ we have

$$
\begin{aligned}
\left|D_{z} A(x, u, z)-D_{z} A(\bar{x}, \bar{u}, \bar{z})\right| & \leq L \chi\left(M,|x-\bar{x}|^{2}+|u-\bar{u}|^{2}+|z-\bar{z}|^{2}\right) \\
& =: L \chi_{M}\left(|x-\bar{x}|^{2}+|u-\bar{u}|^{2}+|z-\bar{z}|^{2}\right) .
\end{aligned}
$$

In this context we now specify the term weak solution:

Definition: $u \in W^{1, p}\left(\Omega, \mathbb{R}^{N}\right)$ is called a weak solution of the Dirichlet problem

$$
\left\{\begin{aligned}
\operatorname{div} A(x, u, D u) & =0 & & \text { in } \Omega \\
u & =g & & \text { on } \partial \Omega
\end{aligned}\right.
$$

if there holds

$$
\int_{\Omega} A(\cdot, u, D u) \cdot D \varphi d x=0 \quad \forall \varphi \in C_{0}^{\infty}\left(\Omega, \mathbb{R}^{N}\right)
$$

and if $u=g$ on $\partial \Omega$ in the sense of traces.

By approximation and taking into account the growth assumption on $A(\cdot, \cdot, \cdot)$ we see that the identity (3) holds for a larger class of test functions, namely all functions $\varphi \in W_{0}^{1, p}\left(\Omega, \mathbb{R}^{N}\right)$.

Our main theorems, the partial regularity in the interior and the characterization of regular (boundary) points, can now be stated: 
Theorem 2.1: Consider $p \in(1,2), \Omega$ a bounded domain in $R^{n}$ with $n \geq 2$ and $u \in W^{1, p}\left(\Omega, \mathbb{R}^{N}\right)$ a weak solution of system (1), where the coefficients $A: \Omega \times \mathbb{R}^{N} \times \mathbb{R}^{n N} \rightarrow \mathbb{R}^{n N}$ fulfill the assumptions (H1), (H2), (H3) and (H4). Then there holds: $u \in C_{l o c}^{1, \alpha}\left(\operatorname{Reg} u, \mathbb{R}^{N}\right)$ and $\operatorname{Sing} u \subseteq \Pi_{1} \cup \Pi_{2}$ with

$$
\begin{aligned}
& \Pi_{1}=\left\{x_{0} \in \Omega: \liminf _{\rho \rightarrow 0^{+}} f_{B_{\rho}\left(x_{0}\right)}\left|V(D u)-(V(D u))_{x_{0}, \rho}\right|^{2} d x>0\right\}, \\
& \Pi_{2}=\left\{x_{0} \in \Omega: \limsup _{\rho \rightarrow 0^{+}}\left(\left|(u)_{x_{0}, \rho}\right|+\left|(V(D u))_{x_{0}, \rho}\right|\right)=\infty\right\} ;
\end{aligned}
$$

In particular, we have $\mathcal{L}^{n}(\operatorname{Sing} u)=0$.

Theorem 2.2: Consider $p \in(1,2)$ and $\Omega \subset \mathbb{R}^{n}$ a bounded domain of class $C^{1, \tau}$ with $n \geq 2$ and some $\tau \in(0,1)$. Let $u \in W^{1, p}\left(\Omega, \mathbb{R}^{N}\right)$ be a weak solution of the system (1), where the coefficients $A: \Omega \times \mathbb{R}^{N} \times \mathbb{R}^{n N} \rightarrow \mathbb{R}^{n N}$ fulfill the assumption (H1), (H2), (H3) and (H4). Assume further the boundary condition (H5). Then if $y \in \operatorname{Reg}_{\partial \Omega} u$ there holds: Du is Hölder-continuous with exponent $\min \{\alpha, \beta, \tau\}$ in a neighbourhood of $y$ in $\bar{\Omega}$ and $\operatorname{Sing}_{\partial \Omega} u \subset \widetilde{\Sigma}_{1} \cup \widetilde{\Sigma}_{2}$ with

$$
\begin{aligned}
& \widetilde{\Sigma}_{1}=\left\{y \in \partial \Omega: \liminf _{\rho \rightarrow 0^{+}} f_{\Omega \cap B_{\rho}(y)}\left|V\left(D_{\nu_{\partial \Omega}(y)}(u-g)\right)-\left(V\left(D_{\nu_{\partial \Omega}(y)}(u-g)\right)\right)_{y, \rho}\right|^{2} d x>0\right\}, \\
& \widetilde{\Sigma}_{2}=\left\{y \in \partial \Omega: \limsup _{\rho \rightarrow 0^{+}} \mid\left(V\left(D_{\nu_{\partial \Omega}(y)}(u-g)\right)_{y, \rho} \mid=\infty\right\} .\right.
\end{aligned}
$$

The main step in the proof of Theorem 2.2 is to prove the result in the model situation of a half-ball. We want to describe briefly how the Dirichlet problem (1) is transformed to a Dirchlet problem on a half-ball. For details we refer to [2], chapter 2.3. Let $z \in \partial \Omega$. Without loss of generality we suppose $z=0$ and $\nu_{\partial \Omega}(z)=e_{n}$. For sufficiently small radius $r>0$ we consider the function $\tilde{v}(y):=u \circ \mathcal{T}^{-1}(y)-g \circ \mathcal{T}^{-1}(y)$ on $B_{r}^{+}$where $\mathcal{T}$ is the function which locally straightens the boundary and $\mathcal{T}^{-1}$ is its inverse. Via the transformation formula we see that $\tilde{v}$ is a solution of

$$
\left\{\begin{aligned}
\operatorname{div} \widetilde{A}(\cdot, \tilde{v}, D \tilde{v}) & =0 & & \text { in } B_{r}^{+} \\
\tilde{v} & =0 & & \text { on } \Gamma_{r}
\end{aligned}\right.
$$

where $\widetilde{A}_{\kappa}^{i}(y, v, z)=A_{\sigma}^{i}\left(\mathcal{T}^{-1}(y), v+\tilde{g}(y),(z+D \tilde{g}(y)) D \mathcal{T}\left(\mathcal{T}^{-1}(y)\right)\right) D_{\sigma} \mathcal{T}^{\kappa}\left(\mathcal{T}^{-1}(y)\right)$ (we sum over repeated indices) are the transformed coefficients. Using assumptions (H1)-(H4) we calculate that $\widetilde{A}$ satisfy structure conditions analogous to (H1)-(H4) with new constants depending on $\|g\|_{C^{1, \beta}},\|\mathcal{T}\|_{C^{1, \tau}},\left\|\mathcal{T}^{-1}\right\|_{C^{1, \tau}}$ and the original ellipticity constant $\nu$ and upper bound $L$. Furthermore, we get as modulus of continuity $\widetilde{\omega}(t)=\min \left\{1, t^{\tilde{\alpha}}\right\}$.

\section{Preliminaries}

In this section we point out some basic and technical results which shall be used throughout the paper. To handle the subquadratic case the $V$ - and the $W$-function will be useful. For all $\xi \in \mathbb{R}^{k}$ and $p>1$ they are defined as

$$
V(\xi)=\left(1+|\xi|^{2}\right)^{\frac{p-2}{4}} \xi \quad \text { and } \quad W(\xi)=\left(1+|\xi|^{2-p}\right)^{-\frac{1}{2}} \xi
$$

The crucial point is their property concerning growth: they behave linearly for $|\xi|$ very small, but grow like $|\xi|^{\frac{p}{2}}$ for $|\xi| \rightarrow \infty$. As shown in [11], Chapter 3, we have

$$
|W(\xi)| \leq|V(\xi)| \leq c(p)|W(\xi)|
$$

with $c(p) \leq \sqrt{2}$ if $p \in(1,2)$. The advantage of $W$ over $V$ is that the function $|W|^{\frac{2}{p}}-$ in contrast to $|V|^{\frac{2}{p}}$ - is convex on $\mathbb{R}^{k}$. Some useful properties of $V$ can be found in [5]: 
Lemma 3.1 ([5], Lemma 2.1): Let $p \in(1,2)$ and $V, W: \mathbb{R}^{k} \rightarrow \mathbb{R}^{k}$ be the functions defined in (4). Then for any $\xi, \eta \in \mathbb{R}^{k}$ and $t>0$ there holds:

(i) $2^{\frac{p-2}{4}} \min \left\{|\xi|,|\xi|^{\frac{p}{2}}\right\} \leq|V(\xi)| \leq \min \left\{|\xi|,|\xi|^{\frac{p}{2}}\right\}$,

(ii) $|V(t \xi)| \leq \max \left\{t, t^{\frac{p}{2}}\right\}|V(\xi)|$,

(iii) $|V(\xi+\eta)| \leq c(p)(|V(\xi)|+|V(\eta)|)$,

(iv) $\quad \frac{p}{2}|\xi-\eta| \leq \frac{|V(\xi)-V(\eta)|}{\left(1+|\xi|^{2}+|\eta|^{2}\right)^{\frac{p-2}{4}}} \leq c(k, p)|\xi-\eta|$,

(v) $\quad|V(\xi)-V(\eta)| \leq c(k, p)|V(\xi-\eta)|$,

(vi) $|V(\xi-\eta)| \leq c(p, M)|V(\xi)-V(\eta)|$, provided $|\eta| \leq M$.

The inequalities (i)-(iii) also hold if we replace $V$ by $W$.

Since $|W|^{2}=\left(|W|^{\frac{2}{p}}\right)^{p}$ is convex and satisfies a $p$-growth condition (see Lemma 3.1 (i)), we may apply [8], Chapter 4, Theorem 2.3 to conclude lower semicontinuity of the mapping $u \mapsto \int_{\Omega}|W(D u)|^{2} d x$ with respect to weak convergence in $W^{1, p}\left(\Omega, \mathbb{R}^{N}\right)$.

Furthermore, we can prove with a similar argument to the proof of [13], Lemma 3:

Lemma 3.2: Let $f: \bar{\Omega} \rightarrow \operatorname{Hom}\left(\mathbb{R}^{n}, \mathbb{R}^{N}\right)$ be a function such that $V \circ f$ is Hölder-continuous with exponent $\alpha \in(0,1)$. Then the function $f$ itself is Hölder-continuous in $\bar{\Omega}$ with the same exponent.

Next we want to consider a $W^{1, p}$-function $u$ in the subquadratic case and prove some inequalities of Sobolev-Poincaré-type, both for the interior and the boundary, which are appropriate for our situation.

Lemma 3.3: Let $p \in(1,2), B_{\rho}\left(x_{0}\right) \subset \mathbb{R}^{n}$ with $n \geq 2$ and set $p^{\sharp}=\frac{2 n}{n-p}$. Moreover, let $V$ and $W$ be the functions defined in (4). Then there exists a constant $c_{s}$ depending only on $n, N$ and $p$ such that for every $u \in W^{1, p}\left(B_{\rho}\left(x_{0}\right), \mathbb{R}^{N}\right)$

$$
\left(f_{B_{\rho}\left(x_{0}\right)}\left|W\left(\frac{u-(u)_{x_{0}, \rho}}{\rho}\right)\right|^{p^{\sharp}} d x\right)^{\frac{1}{p^{\sharp}}} \leq c_{s}\left(f_{B_{\rho}\left(x_{0}\right)}|W(D u)|^{2} d x\right)^{\frac{1}{2}}
$$

and that for every $u \in W_{\Gamma}^{1, p}\left(B_{r}^{+}\left(x_{0}\right), \mathbb{R}^{N}\right)$ with $x_{0} \in \mathbb{R}^{n-1} \times\{0\}$

$$
\left(f_{B_{\rho}^{+}\left(x_{0}\right)}\left|W\left(\frac{u}{\rho}\right)\right|^{p^{\sharp}} d x\right)^{\frac{1}{p^{\sharp}}} \leq c_{s}\left(f_{B_{\rho}^{+}\left(x_{0}\right)}|W(D u)|^{2} d x\right)^{\frac{1}{2}} .
$$

Furthermore, the analogous inequalities hold if we replace $W$ by $V$.

Proof: The proof of the interior estimate can be found in [11], Theorem 2. In the boundary situation we extend $u$ to the whole ball by zero, use Lemma 3.1 and apply Jensen's inequality, and the result follows from the estimate on the full ball in a more or less standard way.

In the next step we will have a closer look at the Poincaré inequality for $u \in W_{\Gamma}^{1, p}\left(B_{R}^{+}, \mathbb{R}^{N}\right)$. We use the fact that $u$ vanishes on $\Gamma$ to show that the integral over $u$ is in fact estimated by the integral of the normal derivative $D_{n} u$ only rather than the full derivative.

Lemma 3.4: For functions $u \in W_{\Gamma}^{1, p}\left(B_{R}^{+}\left(x_{0}\right), \mathbb{R}^{N}\right)$ with $x_{0} \in \mathbb{R}^{n-1} \times\{0\}$ there holds:

$$
\int_{B_{R}^{+}\left(x_{0}\right)}|u|^{p} d x \leq \frac{R^{p}}{p} \int_{B_{R}^{+}\left(x_{0}\right)}\left|D_{n} u\right|^{p} d x .
$$

Proof: We may assume $x_{0}=0$. A function $u(x) \in C^{1}\left(B_{R}^{+}, \mathbb{R}^{N}\right) \cap W_{\Gamma}^{1, p}\left(B_{R}^{+}, \mathbb{R}^{N}\right)$ can be written as

$$
u(x)=u\left(x^{\prime}, x_{n}\right)=u\left(x^{\prime}, x_{n}\right)-u\left(x^{\prime}, 0\right)=\int_{0}^{x_{n}} D_{n} u\left(x^{\prime}, t\right) d t .
$$


Then we conclude using Jensen's inequality and Fubini's theorem:

$$
\begin{aligned}
& \int_{B_{R}^{+}}|u(x)|^{p} d x=\int_{-R}^{R} \int_{-\sqrt{R^{2}-x_{1}^{2}}}^{\sqrt{R^{2}-x_{1}^{2}}} \ldots \int_{0}^{\sqrt{R^{2}-\left|x^{\prime}\right|^{2}}}|u(x)|^{p} d x_{n} d x_{n-1} \ldots d x_{1} \\
& \leq \int_{-R}^{R} \int_{-\sqrt{R^{2}-x_{1}^{2}}}^{\sqrt{R^{2}-x_{1}^{2}}} \ldots \int_{0}^{\sqrt{R^{2}-\left|x^{\prime}\right|^{2}}}\left(x_{n} \int_{0}^{x_{n}}\left|D_{n} u\left(x^{\prime}, t\right)\right| d t\right)^{p} d x_{n} d x_{n-1} \ldots d x_{1} \\
& \leq \int_{-R}^{R} \int_{-\sqrt{R^{2}-x_{1}^{2}}}^{\sqrt{R^{2}-x_{1}^{2}}} \ldots \int_{0}^{\sqrt{R^{2}-\left|x^{\prime}\right|^{2}}} x_{n}^{p-1} \int_{0}^{\sqrt{R^{2}-\left|x^{\prime}\right|^{2}}}\left|D_{n} u\left(x^{\prime}, t\right)\right|^{p} d t d x_{n} d x_{n-1} \ldots d x_{1} \\
& =\frac{R^{p}}{p} \int_{B_{R}^{+}}\left|D_{n} u(x)\right|^{p} d x .
\end{aligned}
$$

For functions $u \in W_{\Gamma}^{1, p}\left(B_{R}^{+}, \mathbb{R}^{N}\right)$ the result is achieved via approximation.

We wish to have an analogous result involving the function $V$. To this end, we need the following lemma:

Lemma 3.5: Let $p \in(1,2)$ and $T: L^{p}\left(B^{+}, \mathbb{R}^{N}\right) \rightarrow L^{p}\left(B^{+}, \mathbb{R}^{N}\right)$ be a bounded, linear operator whose restriction $\left.T\right|_{L^{2}}$ is also a bounded linear operator $L^{2}\left(B^{+}, \mathbb{R}^{N}\right) \rightarrow L^{2}\left(B^{+}, \mathbb{R}^{N}\right)$, i. e., $\|T\|_{L^{2}\left(B^{+}, \mathbb{R}^{N}\right)}:=$ $\left\|\left.T\right|_{L^{2}}\right\|_{L^{2}\left(B^{+}, \mathbb{R}^{N}\right)}<\infty$. Then there exists a constant c depending on $p,\|T\|_{L^{p}\left(B^{+}, \mathbb{R}^{N}\right)},\|T\|_{L^{2}\left(B^{+}, \mathbb{R}^{N}\right)}$ such that for all $f \in L^{p}\left(B^{+}, \mathbb{R}^{N}\right)$ we have

$$
f_{B^{+}}|V(T f)|^{2} d x \leq c f_{B^{+}}|V(f)|^{2} d x .
$$

Proof: The result is shown by a slight modification of the proof of [4], Lemma 2.1.

Lemma 3.6: Let $p \in(1,2)$ and $B_{\rho}^{+}\left(x_{0}\right) \subset \mathbb{R}^{n}$ with $x_{0} \in \mathbb{R}^{n-1} \times\{0\}, n \geq 2$. Then for all $u \in$ $W_{\Gamma}^{1, p}\left(B_{\rho}^{+}\left(x_{0}\right), \mathbb{R}^{N}\right)$ there holds

$$
f_{B_{\rho}^{+}\left(x_{0}\right)}\left|V\left(\frac{u}{\rho}\right)\right|^{2} d x \leq c(p) f_{B_{\rho}^{+}\left(x_{0}\right)}\left|V\left(D_{n} u\right)\right|^{2} d x .
$$

Furthermore, the analogous inequality holds if we replace $W$ by $V$.

Proof: We consider for $f \in C^{1}\left(\overline{B^{+}}, \mathbb{R}^{N}\right)$ the linear operator

$$
T f(x):=\int_{0}^{x_{n}} f\left(x^{\prime}, t\right) d t \quad \text { for } x \in \overline{B^{+}} .
$$

Then we have $\|T f\|_{L^{q}\left(B^{+}, \mathbb{R}^{N}\right)} \leq\|f\|_{L^{q}\left(B^{+}, \mathbb{R}^{N}\right)}$ for $q=2$ and $q=p$. Therefore, we may extend $T$ to a linear operator (called $T$ again) on $L^{q}\left(B^{+}, \mathbb{R}^{N}\right)$. Applying Lemma 3.5 we draw the conclusion:

$$
f_{B^{+}}|V(T f)|^{2} d x \leq c(p) f_{B^{+}}|V(f)|^{2} d x .
$$

If we have that $f$ vanishes on $\Gamma$, the identity $T\left(D_{n} f\right)=f$ holds on $\overline{B^{+}}$; therefore, the estimate of the lemma is proved in the particular situation of $f$ being a continuously differentiable function. The general case follows by approximation in $W^{1, p}\left(\overline{B^{+}}, \mathbb{R}^{N}\right)$ and a standard transformation argument.

\section{$4 \quad$ Linear theory and $\mathcal{A}$-harmonic approximation}

In this section we first provide an a priori estimate for solutions of linear elliptic systems of second order with constant coefficients in the subquadratic case. It is well known that $W^{1,2}$-solutions are smooth up to the boundary. Here we have to use different techniques with the $L^{p}$-theory in a global version as an essential tool to overcome the difficulties arising from the fact that we treat the case $1<p<2$. Secondly we present a suitable $\mathcal{A}$-harmonic approximation lemma. 
We will apply later the a priori estimate for solutions of a homogeneous elliptic systems with constant coefficients $A \in \operatorname{Bil}\left(\mathbb{R}^{n N}\right)$ on half-balls. Therefore, we will consider in what follows the Dirichlet problem

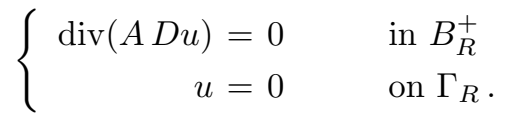

We assume the coefficients $A$ to be bounded and elliptic in the sense of Legendre-Hadamard, i. e., for some $\nu, L>0$ there holds

$$
\begin{array}{lll}
\text { (A1) } & |A(C, \bar{C})| \leq \Lambda|C||\bar{C}| & \forall C, \bar{C} \in \mathbb{R}^{n N}, \\
\text { (A2) } & A(\xi \otimes \eta, \xi \otimes \eta) \geq \nu|\xi|^{2}|\eta|^{2} & \forall \xi \in \mathbb{R}^{N}, \eta \in \mathbb{R}^{n} .
\end{array}
$$

Theorem 4.1 ( $L^{p}$-theory; [14], Satz $\left.\mathbf{1}^{*}\right)$ : Let $\Omega$ be a bounded Lipschitz-domain. Suppose the assumptions (A1) and (A2) hold. Then for any $p>1$ and any $F \in L^{p}\left(\Omega, \operatorname{Hom}\left(\mathbb{R}^{n}, \mathbb{R}^{N}\right)\right)$ there exists exactly one weak solution $u \in W_{0}^{1, p}\left(\Omega, \mathbb{R}^{N}\right)$ of the Dirichlet problem

$$
\int_{\Omega} A(D u, D \varphi) d x=\int_{\Omega} F \cdot D \varphi d x \quad \forall \varphi \in C_{0}^{\infty}\left(\Omega, \mathbb{R}^{N}\right)
$$

and it satisfies

$$
\|u\|_{W_{0}^{1, p}\left(\Omega, \mathbb{R}^{N}\right)} \leq c\|F\|_{L^{p}\left(\Omega, \mathbb{R}^{n N}\right)},
$$

where $c$ is a positive constant depending only on $n, N, p, \frac{\nu}{\Lambda}$ and $\Omega$.

Remark: In fact, in [14] the coefficients do not need to be constant because what is required is only their uniform continuity. Furthermore, the theorem implies that for a homogeneous system the only solution in $W_{0}^{1, p}\left(\Omega, \mathbb{R}^{N}\right)$ is identically zero.

The previous theorem now enables us to prove $C^{\infty}$-regularity for weak solutions $u$ of class $W_{\Gamma}^{1, p}\left(B_{R}^{+}, \mathbb{R}^{N}\right)$.

Theorem 4.2: Let $u \in W_{\Gamma}^{1, p}\left(B_{R}^{+}, \mathbb{R}^{N}\right)$ be a weak solution of the system (5) with constant coefficients $A$ which satisfy conditions (A1) and (A2). Then for any $\rho<\rho_{0}<R$ we have: $u \in C^{\infty}\left(\overline{B_{\rho}^{+}}, \mathbb{R}^{N}\right)$ and

$$
\sup _{B_{\rho}^{+}}\left(\rho^{2}|D u|^{2}+\rho^{4}\left|D^{2} u\right|^{2}\right) \leq c \max \left\{\left(\frac{\rho}{\rho_{0}}\right)^{2},\left(1-\frac{\rho}{\rho_{0}}\right)^{-8 n}\right\} \rho^{2-\frac{2 n}{p}}\left(\int_{B_{\rho_{0}}^{+}}|D u|^{p} d x\right)^{\frac{2}{p}},
$$

where the constant $c$ depends only on $n, N, p$ and $\frac{\nu}{\Lambda}$.

Remark 4.3: In the interior, the $C^{\infty}$-regularity result for weak solutions and the corresponding estimate is easier to derive: since we consider systems with constant coefficients, we can make use of the fact that the smoothed solution is again a solution in the interior. As a consequence, we observe for functions $u \in W^{1,1}\left(B_{\rho}\left(x_{0}\right), \mathbb{R}^{N}\right)$ which are solutions of a linear elliptic system with constant coefficients under conditions (A1) and (A2): $u \in C^{\infty}\left(B_{\rho}\left(x_{0}\right), \mathbb{R}^{N}\right)$ and

$$
\sup _{B_{\rho / 2}\left(x_{0}\right)}\left(|D u|+\rho\left|D^{2} u\right|\right) \leq c f_{B_{\rho}\left(x_{0}\right)}|D u| d x
$$

where the constant $c$ depends only on $n, N$ and $\frac{\nu}{\Lambda}$ (see [11], Lemma 5).

Proof: First we consider a weak solution $u \in W_{\Gamma}^{1,2}\left(B_{R}^{+}, \mathbb{R}^{N}\right)$. By [17], Chapter 10.2, we derive for any radius $r<R$ and any $p>1$ the estimate

$$
\sup _{B_{r}^{+}}\left(r^{2}|D u|^{2}+r^{4}\left|D^{2} u\right|^{2}\right) \leq c \max \left\{\left(\frac{r}{R}\right)^{2},\left(1-\frac{r}{R}\right)^{-8 n}\right\} r^{2-\frac{2 n}{p}}\left(\int_{B_{R}^{+}}\left|D_{n} u\right|^{p} d x\right)^{\frac{2}{p}},
$$

where the constant $c$ depends only on $n, N, p$ and $\frac{\nu}{\Lambda}$. 
Next we want to approximate our solution $u \in W_{\Gamma}^{1, p}\left(B_{R}^{+}, \mathbb{R}^{N}\right)$ by functions $w_{k} \in W_{\Gamma}^{1,2}\left(B_{\rho_{0}}^{+}, \mathbb{R}^{N}\right)$ which are again solutions of appropriate Dirichlet problems. For this purpose we extend $u$ to $B_{R}$ by odd reflection

$$
\tilde{u}(x)=\left\{\begin{array}{cl}
u\left(x^{\prime}, x_{n}\right) & \text { if } x \in B^{+} \cup \Gamma \\
-u\left(x^{\prime},-x_{n}\right) & \text { if } x \in B^{-} .
\end{array}\right.
$$

Let $0<\rho<\rho_{0}<R, \phi \in C_{0}^{\infty}\left(B_{R}\right)$ be a standard mollifier and $\left(\varepsilon_{k}\right)_{k \in \mathbb{N}}$ a positive sequence tending to zero with $\varepsilon_{k} \leq \min \left\{\rho_{0}-\rho, R-\rho_{0}\right\}$ for all $k \in \mathbb{N}$. Let $\phi_{\varepsilon_{k}}$ with $\phi_{\varepsilon_{k}}(x)=\varepsilon_{k}^{-n} \phi\left(\frac{x}{\varepsilon_{k}}\right)$ for $x \in \mathbb{R}^{n}$ be the associated kernels. Then we obtain for the smoothed functions $u_{k}:=\phi_{\varepsilon_{k}} \circ \tilde{u}$ that $u_{k} \in C^{\infty}\left(B_{\rho_{0}}, \mathbb{R}^{N}\right)$, in particular $u_{k} \in W^{1,2}\left(B_{\rho_{0}}, \mathbb{R}^{N}\right)$, and

$$
u_{k}=0 \text { on } \Gamma_{\rho_{0}}, \quad u_{k} \rightarrow u \text { in } W^{1, p}\left(B_{\rho_{0}}^{+}, \mathbb{R}^{N}\right) .
$$

We consider the following Dirichlet problem in $W^{1,2}\left(B_{\rho_{0}}^{+}, \mathbb{R}^{N}\right)$ :

$$
\left\{\begin{aligned}
\int_{B_{\rho_{0}}^{+}} A\left(D w_{k}, D \varphi\right) d x & =0 & & \forall \varphi \in C_{0}^{1}\left(B_{\rho_{0}}^{+}, \mathbb{R}^{N}\right) \\
w_{k} & =u_{k} & & \text { on } B_{\rho_{0}}^{+} .
\end{aligned}\right.
$$

Using Lax-Milgram we obtain the existence of a unique weak solution $w_{k} \in W^{1,2}\left(B_{\rho_{0}}^{+}, \mathbb{R}^{N}\right)$, and the function $v_{k}:=w_{k}-u_{k} \in W_{0}^{1,2}\left(B_{\rho_{0}}^{+}, \mathbb{R}^{N}\right)$ satisfies for all $\varphi \in C_{0}^{1}\left(B_{\rho_{0}}^{+}, \mathbb{R}^{N}\right)$ :

$$
\int_{B_{\rho_{0}}^{+}} A\left(D v_{k}, D \varphi\right) d x=-\int_{B_{\rho_{0}}^{+}} A\left(D u_{k}, D \varphi\right) d x .
$$

From the $L^{p}$-theory, Theorem 4.1, we infer

$$
\int_{B_{\rho_{0}}^{+}}\left|D v_{k}-D v_{j}\right|^{p} d x \leq c \int_{B_{\rho_{0}}^{+}}\left|D u_{k}-D u_{j}\right|^{p} d x
$$

where $c=c\left(n, N, p, \frac{\nu}{\Lambda}, \rho_{0}\right)$. Applying the Poincaré inequality (note that $v_{k}, v_{j}=0$ on $\partial B_{\rho_{0}}^{+}$) we conclude that $\left\{v_{k}\right\}$ is a Cauchy sequence in $W_{0}^{1, p}\left(B_{\rho_{0}}^{+}, \mathbb{R}^{N}\right)$, which converges to a function $v \in W_{0}^{1, p}\left(B_{\rho_{0}}^{+}, \mathbb{R}^{N}\right)$. We used here the trace theorem to infer the zero-boundary values. Therefore, we obtain with (8), the convergence $u_{j} \rightarrow u$ in $W_{0}^{1, p}\left(B_{\rho_{0}}^{+}, \mathbb{R}^{N}\right)$ and (5)

$$
\begin{aligned}
\int_{B_{\rho_{0}}^{+}} A(D v, D \varphi) d x & =\lim _{k \rightarrow \infty} \int_{B_{\rho_{0}}^{+}} A\left(D v_{k}, D \varphi\right) d x \\
& =-\lim _{k \rightarrow \infty} \int_{B_{\rho_{0}}^{+}} A\left(D u_{k}, D \varphi\right) d x=-\int_{B_{\rho_{0}}^{+}} A(D u, D \varphi) d x=0
\end{aligned}
$$

for all functions $\varphi \in C_{0}^{1}\left(B_{\rho_{0}}^{+}, \mathbb{R}^{N}\right)$. Since $v$ vanishes on $\partial B_{\rho_{0}}^{+}$, this implies according to the remark after Theorem 4.1 that $v$ is identically equal to zero.

Using $w_{k}=v_{k}+u_{k}$ it is easily shown that $\left\{w_{k}\right\}$ is a also Cauchy sequence in $W^{1, p}\left(B_{\rho_{0}}^{+}, \mathbb{R}^{N}\right)$ (one needs to keep in mind that $w_{k}=0$ on $\Gamma_{\rho_{0}}$ for the application of the Poincaré inequality in Lemma 3.4). Hence, $w_{k}$ converges to some $w$ in $W^{1, p}\left(B_{\rho_{0}}^{+}, \mathbb{R}^{N}\right)$, and from (7) we see

$$
\int_{B_{\rho_{0}}^{+}} A(D w, D \varphi) d x=0 .
$$

With $v=0$ this yields $w=u$.

Now we have finished the construction of the sequence $\left\{w_{k}\right\}$ converging to $u$ where $w_{k} \in W^{1,2}\left(B_{\rho_{0}}^{+}, \mathbb{R}^{N}\right)$ solves the linear elliptic system $\int_{B_{\rho_{0}}^{+}} A\left(D w_{k}, D \varphi\right) d x=0$ for all $\varphi \in C_{0}^{1}\left(B_{\rho_{0}}^{+}, \mathbb{R}^{N}\right)$. Therefore, we can apply the a priori estimate $(6)$ with $\left(\rho, \rho_{0}\right)$ instead of $(r, R)$ to find

$$
\sup _{B_{\rho}^{+}}\left(\rho^{2}\left|D w_{k}\right|^{2}+\rho^{4}\left|D^{2} w_{k}\right|^{2}\right) \leq c \max \left\{\left(\frac{\rho}{\rho_{0}}\right)^{2},\left(1-\frac{\rho}{\rho_{0}}\right)^{-8 n}\right\} \rho^{2-\frac{2 n}{p}}\left(\int_{B_{\rho_{0}}^{+}}\left|D w_{k}\right|^{p} d x\right)^{\frac{2}{p}} .
$$


In particular, $w_{k}$ is smooth on the ball $\overline{B_{\rho}^{+}}$. Since also the function $w_{k}-w_{j} \in W^{1,2}\left(B_{\rho_{0}}^{+}, \mathbb{R}^{N}\right)$ is a solution of this system, we can apply (9) to this difference, and therefore, we arrive at the conclusion that $\left\{w_{k}\right\}$ is a uniform Cauchy sequence in $C^{2}\left(\overline{B_{\rho}^{+}}, \mathbb{R}^{N}\right)$. Thus there exists $\widetilde{w}$ in $C^{2}\left(\overline{B_{\rho}^{+}}, \mathbb{R}^{N}\right)$ such that

$$
w_{k} \rightarrow \widetilde{w} \text { in } C^{2}\left(\overline{B_{\rho}^{+}}, \mathbb{R}^{N}\right) .
$$

On the other hand we have the convergence $w_{k} \rightarrow u$ in $W^{1, p}\left(B_{\rho_{0}}^{+}, \mathbb{R}^{N}\right)$, and so by the uniqueness of the limit we observe $u=\widetilde{w}$ in $\overline{B_{\rho}^{+}}$. Thus $u \in C^{2}\left(\overline{B_{\rho}^{+}}, \mathbb{R}^{N}\right)$ (in fact it is smooth), and taking the limit in (9) we obtain the desired estimate.

In the second part of this chapter we deal with $\mathcal{A}$-harmonic approximation, which extends the method of harmonic approximation (i. e., approximating with functions solving the Laplace equation) in a natural way to bounded elliptic operators with constant coefficients.

Lemma 4.4 (A-harmonic approximation): Let $\lambda, \Lambda$ be positive constants. Then for any $\varepsilon>0$ there exists $\delta=\delta\left(n, N, p, \frac{\nu}{\Lambda}, \varepsilon\right)$ with the following property: for any bilinear form $\mathcal{A}$ on $\mathbb{R}^{n N}$ which is elliptic in the sense of Legendre-Hadamard with ellipticity constant $\nu$ and upper bound $\Lambda$ and for any $u \in W_{\Gamma}^{1, p}\left(B_{\rho}^{+}\left(x_{0}\right), \mathbb{R}^{N}\right)$ (with some $\rho>0, x_{0} \in \mathbb{R}^{n-1} \times\{0\}$ ) satisfying:

$$
\begin{gathered}
f_{B_{\rho}^{+}\left(x_{0}\right)}|W(D u)|^{2} d x \leq \gamma^{2} \leq 1, \\
\left|f_{B_{\rho}^{+}\left(x_{0}\right)} \mathcal{A}(D u, D \varphi) d x\right| \leq \delta \gamma \sup _{B_{\rho}^{+}\left(x_{0}\right)}|D \varphi| \quad \forall \varphi \in C_{0}^{1}\left(B_{\rho}^{+}\left(x_{0}\right), \mathbb{R}^{N}\right),
\end{gathered}
$$

there exists an $\mathcal{A}$-harmonic function $h \in W_{\Gamma}^{1, p}\left(B_{\rho / 2}^{+}\left(x_{0}\right), \mathbb{R}^{N}\right)$, i. e., $\int_{B_{\rho / 2}^{+}\left(x_{0}\right)} \mathcal{A}(D h, D \varphi) d x=0$ for all functions $\varphi \in C_{0}^{1}\left(B_{\rho / 2}^{+}\left(x_{0}\right), \mathbb{R}^{N}\right)$, which satisfies

$$
f_{B_{\rho / 2}^{+}\left(x_{0}\right)}\left|W\left(\frac{u-\gamma h}{\rho}\right)\right|^{2} d x \leq \gamma^{2} \varepsilon \quad \text { and } \quad f_{B_{\rho / 2}^{+}\left(x_{0}\right)}|W(D h)|^{2} d x \leq 2^{n+2} .
$$

Remark 4.5: An analogous result in the interior can be found in [11], Lemma 6. Instead of going to balls of half radius as above, it was shown that there exists an $\mathcal{A}$-harmonic function $h$ on the full ball $B_{\rho}\left(x_{0}\right)$ which satisfies an estimate equivalent to $(10)$ on $B_{\rho}\left(x_{0}\right)$.

Proof: For the proof of this lemma we combine ideas from [11], Lemma 6, which treats the subquadratic situation, with ideas of [20], Lemma 2.1, which treats the boundary in the quadratic case. Without loss of generality we may assume $x_{0}=0$ and $\rho=1$. The general case follows by a rescaling argument.

If the conclusion of the lemma were false, we could find $\varepsilon>0$ and sequences $\left\{\mathcal{A}_{k}\right\}$ of bilinear forms on $\mathbb{R}^{n N}$ with uniform ellipticity constant $\nu$ and uniform upper bound $\Lambda,\left\{f_{k}\right\}$ with $f_{k} \in W_{\Gamma}^{1, p}\left(B^{+}, \mathbb{R}^{N}\right)$ and $\left\{\gamma_{k}\right\}$ with $\gamma_{k} \in(0,1]$ such that

$$
f_{B^{+}}\left|W\left(D f_{k}\right)\right|^{2} d x \leq \gamma_{k}^{2} \leq 1 \text { and }\left|f_{B^{+}} \mathcal{A}\left(D f_{k}, D \varphi\right) d x\right| \leq \frac{\gamma_{k}}{k} \sup _{B^{+}}|D \varphi|
$$

for all $\varphi \in C_{0}^{1}\left(B^{+}, \mathbb{R}^{N}\right)$, but

$$
f_{B_{1 / 2}^{+}}\left|W\left(f_{k}-\gamma_{k} h_{k}\right)\right|^{2} d x>\gamma_{k}^{2} \varepsilon
$$

for all $h_{k} \in \mathcal{H}_{k}:=\left\{f \in W_{\Gamma}^{1, p}\left(B_{1 / 2}^{+}, \mathbb{R}^{N}\right), f\right.$ is $\mathcal{A}_{k}$-harmonic on $B_{1 / 2}^{+}$with $\left.f_{B_{1 / 2}^{+}}|W(D f)|^{2} d x \leq 2^{n+2}\right\}$.

Defining $g_{k}:=\frac{f_{k}}{\gamma_{k}}$ we obtain by $(11)$ :

$$
\left|f_{B^{+}} \mathcal{A}\left(D g_{k}, D \varphi\right) d x\right| \leq \frac{1}{k} \sup _{B^{+}}|D \varphi| \quad \forall \varphi \in C_{0}^{1}\left(B^{+}, \mathbb{R}^{N}\right)
$$


and according to Lemma $3.1(\mathrm{i})$ we see that the $L^{p}$-norm of $D g_{k}$ is bounded on $B^{+}$:

$$
f_{B^{+}}\left|D g_{k}\right|^{p} d x \leq \frac{c(p)}{\left|B^{+}\right| \gamma_{k}^{p}}\left(\int_{B^{+}}\left|W\left(D f_{k}\right)\right|^{2} d x+\int_{B^{+}}\left|W\left(D f_{k}\right)\right|^{p} d x\right) \leq c(p)\left(\gamma_{k}^{2-p}+1\right) .
$$

Applying the Poincaré inequality from Lemma 3.4 to every $g_{k}\left(g_{k}=0\right.$ on $\left.\Gamma\right)$ immediately implies that the sequence $\left\{g_{k}\right\}$ is bounded in $W^{1, p}\left(B^{+}, \mathbb{R}^{N}\right)$ by a constant $c(n, p)$. Hence, passing to a subsequence (also labelled with $k$ ), this yields the existence of $g \in W^{1, p}\left(B^{+}, \mathbb{R}^{N}\right), \mathcal{A} \in \operatorname{Hom}\left(\mathbb{R}^{n}, \mathbb{R}^{N}\right)$ and $\gamma \in[0,1]$ such that there holds

$$
\begin{array}{ll}
g_{k} \rightarrow g & \text { weakly in } W^{1, p}\left(B^{+}, \mathbb{R}^{N}\right), \\
g_{k} \rightarrow g & \text { strongly in } L^{p}\left(B^{+}, \mathbb{R}^{N}\right) \text { and a.e. in } B^{+}, \\
\mathcal{A}_{k} \rightarrow \mathcal{A} & \text { in } \mathbb{R}^{n N}, \\
\gamma_{k} \rightarrow \gamma & \text { monotone. }
\end{array}
$$

Indeed, by the trace theorem, we have $g=0$ on $\Gamma$. Using the lower semicontinuity of $u \mapsto \int_{B^{+}}|W(D u)|^{2} d x$ with respect to weak convergence in $W^{1, p}\left(B^{+}, \mathbb{R}^{N}\right)$, Lemma 3.1 (ii) and (11), we deduce:

$$
f_{B^{+}}|W(D g)|^{2} d x \leq \liminf _{k \rightarrow \infty} f_{B^{+}}\left|W\left(\frac{D f_{k}}{\gamma_{k}}\right)\right|^{2} d x \leq 1
$$

Furthermore, we deduce as in [11], Lemma 6 via the weak convergence of $g_{k} \rightarrow g$ in $W^{1, p}$, the convergence of $\mathcal{A}_{k} \rightarrow \mathcal{A}$ and (13): $g$ is $\mathcal{A}$-harmonic on $B^{+}$, meaning that we have $f_{B^{+}} \mathcal{A}(D g, D \varphi) d x=0$ for all $\varphi \in C_{0}^{1}\left(B^{+}, \mathbb{R}^{N}\right)$. Since $g \in W_{\Gamma}^{1, p}\left(B^{+}, \mathbb{R}^{N}\right)$ is a solution of a linear system with constant coefficients, Theorem 4.2 now allows us to observe that $g$ is smooth on any smaller half-ball $\overline{B_{\rho}^{+}}$with $0<\rho<1$.

We now consider the Dirichlet problem $\left(D_{k}\right)$ given by

$$
\left\{\begin{aligned}
\int_{B_{1 / 2}^{+}} \mathcal{A}_{k}(D v, D \varphi) d x & =0 & & \forall \varphi \in C_{0}^{1}\left(B_{1 / 2}^{+}, \mathbb{R}^{N}\right) \\
v & =g & & \text { on } \partial B_{1 / 2}^{+} .
\end{aligned}\right.
$$

By Lax-Milgram theorem there exists a unique solution $v_{k} \in W_{\Gamma}^{1,2}\left(B_{1 / 2}^{+}, \mathbb{R}^{N}\right)$. Testing the system $\left(D_{k}\right)$ with $v_{k}-g$ and using the strong Legendre-Hadamard condition for each bilinear form $\mathcal{A}_{k}$, the $\mathcal{A}_{k^{-}}$ harmonicity of $v_{k}$, the $\mathcal{A}$-harmonicity of $g$ and Hölder's inequality, we see that:

$$
\nu f_{B_{1 / 2}^{+}}\left|D v_{k}-D g\right|^{2} d x \leq\left|\mathcal{A}-\mathcal{A}_{k}\right| \sup _{B_{1 / 2}^{+}}|D g|\left(f_{B_{1 / 2}^{+}}\left|D v_{k}-D g\right|^{2} d x\right)^{1 / 2} .
$$

As $g$ is smooth on $\overline{B_{1 / 2}^{+}}$and thus $\sup _{B_{1 / 2}^{+}}|D g|$ is bounded, the convergence $\mathcal{A}_{k} \rightarrow \mathcal{A}$ yields that the right hand side of the last inequality vanishes as $k \rightarrow \infty$. Via the Poincaré inequality we infer

$$
v_{k} \rightarrow g \text { strongly in } W_{\Gamma}^{1,2}\left(B_{1 / 2}^{+}, \mathbb{R}^{N}\right)
$$

and therefore, strong convergence $V\left(D v_{k}\right) \rightarrow V(D g)$ in $L^{2}\left(B_{1 / 2}^{+}, \mathbb{R}^{N}\right)$ follows from Lemma $3.1(\mathrm{v})$, (i):

$$
f_{B_{1 / 2}^{+}}\left|V\left(D v_{k}\right)-V(D g)\right|^{2} d x \leq c f_{B_{1 / 2}^{+}}\left|V\left(D v_{k}-D g\right)\right|^{2} d x \leq c(n, N, p) f_{B_{1 / 2}^{+}}\left|D v_{k}-D g\right|^{2} d x .
$$

Then we conclude for $k \rightarrow \infty$ via (14)

$$
f_{B_{1 / 2}^{+}}\left|W\left(D v_{k}\right)\right|^{2} d x \leq f_{B_{1 / 2}^{+}}\left|V\left(D v_{k}\right)\right|^{2} d x \longrightarrow f_{B_{1 / 2}^{+}}|V(D g)|^{2} d x \leq 2^{n+1} .
$$

This yields $f_{B_{1 / 2}^{+}}\left|W\left(D v_{k}\right)\right|^{2} d x \leq 2^{n+2}$ for $k$ sufficiently large.

We have proved so far that the functions $v_{k} \in W_{\Gamma}^{1, p}\left(B_{1 / 2}^{+}, \mathbb{R}^{N}\right)$ belong to $\mathcal{H}_{k}$ for $k$ sufficiently large. In what follows we show that they are suitable to produce a contradiction to (12). We proceed similar to [11], Lemma 6, but for the convenience of the reader we outline the procedure as our situation is less 
complicated (we allow half-balls of half radius for the estimate (10)). Let $p^{\sharp}:=\frac{2 n}{n-p}>2$. Then there exists $t \in\left(0, \frac{1}{2}\right)$ such that $1=2 t+2(1-t) \frac{1}{p^{\sharp}}$, and we can estimate via Hölder's inequality:

$$
f_{B_{1 / 2}^{+}}\left|W\left(f_{k}-\gamma_{k} v_{k}\right)\right|^{2} d x \leq\left(f_{B_{1 / 2}^{+}}\left|W\left(f_{k}-\gamma_{k} v_{k}\right)\right| d x\right)^{2 t}\left(f_{B_{1 / 2}^{+}}\left|W\left(f_{k}-\gamma_{k} v_{k}\right)\right|^{p^{\sharp}} d x\right)^{\frac{2(1-t)}{p^{\sharp}}} .
$$

The first term of the right hand side of (16) is calculated by Lemma 3.1 (i):

$$
\begin{aligned}
\gamma_{k}^{-2 t}\left(f_{B_{1 / 2}^{+}}\left|W\left(f_{k}-\gamma_{k} v_{k}\right)\right| d x\right)^{2 t} & \leq \gamma_{k}^{-2 t}\left(f_{B_{1 / 2}^{+}}\left|f_{k}-\gamma_{k} v_{k}\right| d x\right)^{2 t} \\
& \leq\left(f_{B_{1 / 2}^{+}}\left|g_{k}-g\right| d x\right)^{2 t}+\left(f_{B_{1 / 2}^{+}}\left|g-v_{k}\right| d x\right)^{2 t} \stackrel{k \rightarrow \infty}{\longrightarrow} 0,
\end{aligned}
$$

the convergence being a consequence of the strong convergence $g_{k} \rightarrow g$ in $L^{p}\left(B^{+}, \mathbb{R}^{N}\right)$ and $v_{k} \rightarrow g$ in $L^{2}\left(B^{+}, \mathbb{R}^{N}\right)$ in $(15)$. For the second term on the right hand side of $(16)$ we use the Sobolev-Poincaré inequality from Lemma 3.3, Lemma 3.1 (iii), (i) and (11):

$$
\begin{aligned}
& \left(f_{B_{1 / 2}^{+}}\left|W\left(f_{k}-\gamma_{k} v_{k}\right)\right|^{p^{\sharp}} d x\right)^{\frac{2(1-t)}{p^{\sharp}}} \leq c_{s}(n, N, p)\left(f_{B_{1 / 2}^{+}}\left|W\left(D f_{k}-\gamma_{k} D v_{k}\right)\right|^{2} d x\right)^{1-t} \\
& \leq c(n, N, p)\left(f_{B_{1 / 2}^{+}}\left(\left|W\left(D f_{k}\right)\right|^{2}+\left|W\left(\gamma_{k}\left(D v_{k}-D g\right)\right)\right|^{2}+\left|W\left(\gamma_{k} D g\right)\right|^{2}\right) d x\right)^{1-t} \\
& \leq c(n, N, p)\left(\gamma_{k}^{2}+\gamma_{k}^{2} f_{B_{1 / 2}^{+}}\left|D v_{k}-D g\right|^{2} d x+f_{B_{1 / 2}^{+}}\left|W\left(\gamma_{k} D g\right)\right|^{2} d x\right)^{1-t} .
\end{aligned}
$$

For the last term we argue exactly as in [11], estimate (47): we distinguish the cases that $\left\{\gamma_{k}\right\}$ is, in turn, nondecreasing or nonincreasing, and derive (for $k$ sufficiently large):

$$
f_{B_{1 / 2}^{+}}\left|W\left(\gamma_{k} D g\right)\right|^{2} d x \leq 2^{n+1} \gamma_{k}^{2}
$$

using the weak convergence $\gamma_{k} \frac{D f_{l}}{\gamma_{l}} \rightarrow \gamma_{k} D g$ in $L^{p}\left(B^{+}, \mathbb{R}^{N}\right)$, the lower semicontinuity with respect to weak convergence in $W^{1, p}\left(B_{1 / 2}^{+}, \mathbb{R}^{N}\right)$ of $u \mapsto \int_{B_{1 / 2}^{+}}|W(D u)|^{2} d x$, Lemma 3.1 and the estimate (11). Combined with the estimate (18) and the convergence in (15), this gives

$$
\left(f_{B_{1 / 2}^{+}}\left|W\left(f_{k}-\gamma_{k} v_{k}\right)\right|^{p^{\sharp}} d x\right)^{\frac{2(1-t)}{p^{\sharp}}} \leq c(n, N, p) \gamma_{k}^{2(1-t)} .
$$

From the decomposition (16) and the convergence in (17) we obtain altogether

$$
f_{B_{1 / 2}^{+}}\left|W\left(f_{k}-\gamma_{k} v_{k}\right)\right|^{2} d x \leq \varepsilon \gamma_{k}^{2}
$$

for $k$ sufficiently large, which is the desired contradiction to (12). This finishes the proof of the lemma. $\square$

\section{A Caccioppoli-type inequality}

The first step in proving a regularity theorem for solutions $u$ of elliptic systems is to establish a suitable reverse-Poincaré or Caccioppoli inequality. This means that the derivative $D u$ is essentially controlled by the solution $u$ itself on a larger domain (or, in our model situation, on a larger half-ball).

Lemma 5.1 (Caccioppoli inequality): Let $u \in W_{\Gamma}^{1, p}\left(B^{+}, \mathbb{R}^{N}\right)$ be a weak solution of the homogeneous system

$$
\operatorname{div} A(\cdot, u, D u)=0 \quad \text { in } B^{+}
$$


where the coefficients A satisfy the assumptions (H1), (H2), (H3) and (H4). Let $M>0$. Then for any fixed $\xi \in \mathbb{R}^{N}$ with $|\xi| \leq M, x_{0} \in \Gamma$ and $\rho<1-\left|x_{0}\right|$ there holds:

$$
f_{B_{\rho / 2}^{+}\left(x_{0}\right)}\left|V\left(D u-\xi \otimes e_{n}\right)\right|^{2} d x \leq c_{c a c c}\left(f_{B_{\rho}^{+}\left(x_{0}\right)}\left|V\left(\frac{u-\xi x_{n}}{\rho}\right)\right|^{2} d x+\rho^{2 \alpha}\right)
$$

for a constant $c_{\text {cacc }}$ depending only on $n, p, \frac{L}{\nu}, M$ and $K(M)$.

Remark 5.2: In the interior, on the right hand side of a Caccioppoli inequality, we usually need a function with mean value zero. Thus we show in this setting for any $\mu \in \mathbb{R}^{N}, \Upsilon \in \mathbb{R}^{n N}$ with $|\mu|,|\Upsilon| \leq M$ the following version:

$$
f_{B_{\rho / 2}\left(x_{0}\right)}|V(D u)-V(\Upsilon)|^{2} d x \leq \widehat{c}_{c a c c}\left(f_{B_{\rho}\left(x_{0}\right)}\left|V\left(\frac{u(x)-\mu-\Upsilon\left(x-x_{0}\right)}{\rho}\right)\right|^{2} d x+\rho^{2 \alpha}\right),
$$

where $\widehat{c}_{c a c c}$ depends only on $n, N, p, \frac{L}{\nu}, M$ and $K(2 M)$.

Proof: We consider a cut-off function $\eta \in C_{0}^{\infty}\left(B_{\frac{\rho}{2}}\left(x_{0}\right),[0,1]\right)$, satisfying $\eta \equiv 1$ on $B_{\frac{\rho}{2}}\left(x_{0}\right)$ and $|\nabla \eta| \leq \frac{4}{\rho}$. Since $u \in W_{\Gamma}^{1, p}\left(B_{\rho}^{+}, \mathbb{R}^{N}\right)$, the function $u-\xi \cdot x_{n}$ belongs as well to $W_{\Gamma}^{1, p}\left(B_{\rho}^{+}, \mathbb{R}^{N}\right)$, and we can test the system with $\varphi=\eta^{2}\left(u-\xi x_{n}\right)$. Abbreviating $X=\xi \otimes e_{n}$ we have

$$
\begin{aligned}
0 & =f_{B_{\rho}^{+}\left(x_{0}\right)} A(\cdot, u, D u) \cdot D \varphi d x \\
& =f_{B_{\rho}^{+}\left(x_{0}\right)} A(\cdot, u, D u) \cdot(D u-X) \eta^{2} d x+f_{B_{\rho}^{+}\left(x_{0}\right)} A(\cdot, u, D u) \cdot\left(\left(u-\xi x_{n}\right) \otimes \nabla \eta\right) 2 \eta d x,
\end{aligned}
$$

and finally (taking into account that $A\left(x_{0}, 0, X\right)$ is constant):

$$
\begin{aligned}
& f_{B_{\rho}^{+}\left(x_{0}\right)}[A(\cdot, u, D u)-A(\cdot, u, X)] \cdot(D u-X) \eta^{2} d x \\
& =-f_{B_{\rho}^{+}\left(x_{0}\right)} A(\cdot, u, X) \cdot(D u-X) \eta^{2} d x-2 f_{B_{\rho}^{+}\left(x_{0}\right)} A(\cdot, u, D u) \cdot\left(\left(u-\xi x_{n}\right) \otimes \nabla \eta\right) \eta d x \\
& =-2 f_{B_{\rho}^{+}\left(x_{0}\right)}[A(\cdot, u, D u)-A(\cdot, u, X)] \cdot\left(\left(u-\xi x_{n}\right) \otimes \nabla \eta\right) \eta d x \\
& \quad-f_{B_{\rho}^{+}\left(x_{0}\right)}\left[A(\cdot, u, X)-A\left(\cdot, \xi x_{n}, X\right)\right] \cdot D \varphi d x-f_{B_{\rho}^{+}\left(x_{0}\right)}\left[A\left(\cdot, \xi x_{n}, X\right)-A\left(x_{0}, 0, X\right)\right] \cdot D \varphi d x \\
& =:-f_{B_{\rho}^{+}\left(x_{0}\right)}(2 I+I I+I I I) d x
\end{aligned}
$$

with the obvious labelling. The ellipticity condition (H3) allows us to compute for the left-hand side of (19):

$$
\begin{aligned}
& f_{B_{\rho}^{+}\left(x_{0}\right)}[A(\cdot, u, D u)-A(\cdot, u, X)] \cdot(D u-X) \eta^{2} d x \\
& =f_{B_{\rho}^{+}\left(x_{0}\right)} \int_{0}^{1} D_{z} A(\cdot, u, X+t(D u-X))(D u-X, D u-X) \eta^{2} d t d x \\
& \geq f_{B_{\rho}^{+}\left(x_{0}\right)} \int_{0}^{1} \nu\left(1+|X+t(D u-X)|^{2}\right)^{\frac{p-2}{2}}|D u-X|^{2} \eta^{2} d t d x \\
& \geq \nu c^{-1}(p, M) f_{B_{\rho}^{+}\left(x_{0}\right)}|V(D u-X)|^{2} \eta^{2} d x .
\end{aligned}
$$

To estimate the terms $I, I I$ and $I I I$ one by one, we decompose the half-ball:

$$
B_{(\leq)(>)}:=B_{\rho}^{+}\left(x_{0}\right) \cap\{x:|D u(x)-X| \leq 1\} \cap\left\{x:\left|\frac{u(x)-\xi x_{n}}{\rho}\right|>1\right\},
$$


where other combinations involving $>$ and $\leq$ are defined analogously. On these sets we can use Lemma 3.1 (i) because for any $\zeta \in \mathbb{R}^{k}$ there holds:

$$
\begin{array}{ll}
\text { if }|\zeta| \leq 1: & \min \left\{|\zeta|^{2},|\zeta|^{p}\right\}=|\zeta|^{2} \leq \sqrt{2}|V(\zeta)|^{2} \\
\text { if }|\zeta|>1: & \min \left\{|\zeta|^{2},|\zeta|^{p}\right\}=|\zeta|^{p} \leq \sqrt{2}|V(\zeta)|^{2}
\end{array}
$$

Keeping in mind that $\rho<1$ and using Young's inequality $a \cdot b \leq \frac{q-1}{q} \varepsilon a^{\frac{q}{q-1}}+\frac{1}{q} \varepsilon^{1-q} b^{q}$ (for $a, b, \varepsilon \geq 0$ and $q>1$ ), we can now use the given assumptions on the coefficients $A$ to estimate the various terms: for term $I$ we apply condition (H2) if $x \in B_{(\leq)(\cdot)}$ and condition (H1) if $x \in B_{(>)(\cdot)}$ to conclude

$$
2 I \leq \varepsilon|V(D u-X)|^{2} \eta^{2}+c(p, M)\left(L^{2} \varepsilon^{-1}+L^{p} \varepsilon^{1-p}\right)\left|V\left(\frac{u-\xi x_{n}}{\rho}\right)\right|^{2} .
$$

For the remaining term $I I$ and $I I I$ we use condition (H4) and the fact that we have $\left|u-\xi x_{n}\right| \leq \rho$ in the set $B_{(\cdot)(\leq)}$ to find in a standard way

$$
\begin{aligned}
I I+I I I \leq & 2 \varepsilon|V(D u-X)|^{2} \eta^{2}+c(p, M) K(M)^{\frac{p}{p-1}}\left(L+L^{\frac{p}{p-1}} \varepsilon^{\frac{1}{1-p}}\right)\left|V\left(\frac{u-\xi x_{n}}{\rho}\right)\right|^{2} \\
& +c(p, M) K(M)^{\frac{p}{p-1}}\left(L+L^{\frac{p}{p-1}} \varepsilon^{\frac{1}{1-p}}+L^{2} \varepsilon^{-1}\right) \rho^{2 \alpha} .
\end{aligned}
$$

For detailed calculations we refer to [2], chapter 5. Combining (19), (20), (22) and (23), we infer

$$
\begin{aligned}
& \left(\nu c^{-1}(p, M)-3 \varepsilon\right) f_{B_{\rho}^{+}\left(x_{0}\right)}|V(D u-X)|^{2} \eta^{2} d x \\
& \leq c(p, M) K(M)^{\frac{p}{p-1}}\left(L^{2} \varepsilon^{-1}+L^{p} \varepsilon^{1-p}+L+L^{\frac{p}{p-1}} \varepsilon^{\frac{1}{1-p}}\right) f_{B_{\rho}^{+}\left(x_{0}\right)}\left|V\left(\frac{u-\xi x_{n}}{\rho}\right)\right|^{2} d x \\
& \quad+c(p, M) K(M)^{\frac{p}{p-1}}\left(L+L^{\frac{p}{p-1}} \varepsilon^{\frac{1}{1-p}}+L^{2} \varepsilon^{-1}\right) \rho^{2 \alpha} .
\end{aligned}
$$

Taking $\varepsilon=\frac{1}{6} \nu c^{-1}(p, M)$ and dividing both sides by $\frac{1}{2} \nu c^{-1}(p, M)$, we obtain the desired estimate.

\section{An excess-decay estimate}

For any half-ball $B_{\rho}^{+}(y) \subset \subset B^{+}$with $y \in \Gamma$, a fixed function $u \in W_{\Gamma}^{1, p}\left(B^{+}, \mathbb{R}^{N}\right)$ and $\xi \in \mathbb{R}^{N}$ we define the excess function by

$$
\Phi(y, \rho, \xi):=\left(f_{B_{\rho}^{+}(y)}\left|V\left(D u-\xi \otimes e_{n}\right)\right|^{2} d x\right)^{\frac{1}{2}} .
$$

In this section we consider a solution $u \in W_{\Gamma}^{1, p}\left(B^{+}, \mathbb{R}^{N}\right)$ of the system

$$
\operatorname{div} A(\cdot, u, D u)=0 \quad \text { in } B^{+} .
$$

We will show that the function $u-\xi x_{n}$ is approximately $\mathcal{A}$-harmonic for some constant coefficients $\mathcal{A}$ which are derived from the original coefficients $A$. Application of Lemma 4.4 below will then yield the existence of an $\mathcal{A}$-harmonic function, which is on the one hand comparable via the function $W$ to the function $\left(u-\xi x_{n}\right)$ in the $L^{2}$-sense, and for which we have, on the other hand, already proved good a priori estimates.

Lemma 6.1 (Approximate $\mathcal{A}$-harmonicity): Let $u \in W_{\Gamma}^{1, p}\left(B^{+}, \mathbb{R}^{N}\right)$ be a weak solution of (24), where conditions (H2) and (H4) are satisfied. Then for any $M>0$ there exists a constant $c_{a}=$ $L c(p, M, K(M))$ such that for every half-ball $B_{\rho}^{+}(y) \subset \subset B^{+}, y \in \Gamma$, and every $\xi \in \mathbb{R}^{N}$ with $|\xi| \leq M$ we have:

$$
\left|f_{B_{\rho}^{+}(y)} D_{z} A\left(y, 0, \xi \otimes e_{n}\right)\left(D u-\xi \otimes e_{n}, D \varphi\right) d x\right| \leq c_{a}\left[\Phi^{2}+\rho^{\alpha}+\chi_{M}\left(\Phi^{2}\right) \Phi\right] \sup _{B_{\rho}^{+}(y)}|D \varphi|
$$

for all $\varphi \in C_{0}^{\infty}\left(B_{\rho}^{+}(y), \mathbb{R}^{N}\right)$. Here we have abbreviated $\Phi(y, \rho, \xi)$ by $\Phi$. 
Proof: We use again the abbreviation $X=\xi \otimes e_{n}$. We assume initially $\sup _{B_{\rho}^{+}(y)}|D \varphi| \leq 1$. Since $A(y, 0, X)$ is constant and $\operatorname{div} A(\cdot, u, D u)=0$ we see:

$$
\begin{aligned}
& f_{B_{\rho}^{+}(y)} \int_{0}^{1} D_{z} A(y, 0, x+t(D u-X)) d t(D u-X, D \varphi) d x \\
& =f_{B_{\rho}^{+}(y)}[A(y, 0, D u)-A(y, 0, x)] \cdot D \varphi d x=f_{B_{\rho}^{+}(y)}[A(y, 0, D u)-A(\cdot, u, D u)] \cdot D \varphi d x,
\end{aligned}
$$

and thus

$$
\begin{aligned}
& \left|f_{B_{\rho}^{+}(y)} D_{z} A(y, 0, X)(D u-x, D \varphi) d x\right| \\
& \leq f_{B_{\rho}^{+}(y)}\left(\left|\int_{0}^{1}\left[D_{z} A(y, 0, x)-D_{z} A(y, 0, x+t(D u-x))\right] d t\right||(D u-x)|\right. \\
& \quad \quad+|A(y, 0, D u)-A(x, X(x-y), D u)|+|A(x, X(x-y), D u)-A(x, u, D u)|) d x \\
& =f_{B_{\rho}^{+}(y)}(I+I I+I I I) d x
\end{aligned}
$$

with the obvious labelling. In all the estimates of the terms $I, I I$ and $I I I$ below we will distinguish the cases $|D u-X| \leq 1,|D u-X|>1$ and use (21):

Estimate for $I$ : On the set $B_{\rho}^{+}(y) \cap\{|D u-X|>1\}$ from the boundedness of $D_{z} A$ in (H2) we get

$$
I \leq 2 L|D u-X| \leq 2 L|D u-X|^{p} \leq 2 \sqrt{2} L|V(D u-X)|^{2} .
$$

On the complement, we use the existence of the modulus of continuity $\chi_{M}$ (see (2)) to conclude

$$
\begin{aligned}
I & \leq \int_{0}^{1}\left|D_{z} A(y, 0, X)-D_{z} A(y, 0, X+t(D u-X))\right| d t|D u-X| \\
& \leq L \chi_{M}\left(\sqrt{2}|V(D u-X)|^{2}\right) 2^{\frac{1}{4}}|V(D u-X)| .
\end{aligned}
$$

Since $\chi_{M}^{2}$ is concave and monotone nondecreasing, we can apply Hölder's and Jensen's inequality (note that we have $\chi_{M}^{2}(c t) \leq c \chi_{M}^{2}(t)$ for $\left.c \geq 1\right)$ to arrive at

$$
\begin{aligned}
\frac{1}{\left|B_{\rho}^{+}(y)\right|} \int_{B_{\rho}^{+}(y) \cap\{|D u-x| \leq 1\}} I d x & \leq 2^{\frac{1}{4}} L f_{B_{\rho}^{+}(y)} \chi_{M}\left(\sqrt{2}|V(D u-X)|^{2}\right)|V(D u-X)| d x \\
& \leq \sqrt{2} L \chi_{M}\left(\Phi^{2}(y, \rho, \xi)\right) \Phi(y, \rho, \xi) .
\end{aligned}
$$

Therefore, we achieve for the first integral:

$$
f_{B_{\rho}^{+}(y)} I d x \leq 2 \sqrt{2} L \Phi^{2}(y, \rho, \xi)+\sqrt{2} L \chi_{M}\left(\Phi^{2}(y, \rho, \xi)\right) \Phi(y, \rho, \xi) .
$$

Estimate for II: By assumption (H4) we have:

$$
\begin{aligned}
I I & \leq L K(|X|) \omega(|x-y|+|X||x-y|)\left(1+|D u|^{2}\right)^{\frac{p-1}{2}} \\
& \leq L K(M) c(M) \rho^{\alpha}\left(1+|D u-X|^{p-1}\right) .
\end{aligned}
$$

Hence, distinguishing the cases $B_{\rho}^{+}(y) \cap\{|D u-X|>1\}$ (note here $\rho \leq 1$ ) and $B_{\rho}^{+}(y) \cap\{|D u-X| \leq 1\}$, we obtain for the second integral the estimate:

$$
f_{B_{\rho}^{+}(y)} I I d x \leq L K(M) c(M)\left(\Phi^{2}(y, \rho, \xi)+\rho^{\alpha}\right) .
$$

Estimate for III: Note that $X(x-y)=\xi\left(x_{n}-y_{n}\right)=\xi x_{n}$. Similar to the estimate for term $I I$, using (H4), we see:

$$
I I I \leq L K(M) 2(1+M) \omega\left(\left|u-\xi x_{n}\right|\right)\left(1+|D u-X|^{p-1}\right) .
$$


Since $\omega(t) \leq 1$ this implies on $B_{\rho}^{+}(y) \cap\{|D u-X|>1\}$ :

$$
I I I \leq L K(M) c(M)|D u-\mathcal{X}|^{p} \leq L K(M) c(M)|V(D u-X)|^{2} .
$$

On $B_{\rho}^{+}(y) \cap\{|D u-X| \leq 1\}$ we first distinguish the cases $\left\{\left|\frac{u-\xi x_{n}}{\rho}\right| \leq 1\right\},\left\{\left|\frac{u-\xi x_{n}}{\rho}\right|>1\right\}$ to find $\left|u-\xi x_{n}\right|^{\alpha} \leq \rho^{\alpha}+\sqrt{2}\left|V\left(\frac{u-\xi x_{n}}{\rho}\right)\right|^{2}$. Therefore, we derive on the set $B_{\rho}^{+}(y) \cap\{|D u-X| \leq 1\}$

$$
I I I \leq L K(M) c(M)\left(\rho^{\alpha}+\left|V\left(\frac{u-\xi x_{n}}{\rho}\right)\right|^{2}\right) .
$$

Since $u-\xi x_{n}$ vanishes on $\Gamma$, in particular on $\Gamma_{\rho}(y)$, we can apply the Poincaré inequality from Lemma 3.6 to deduce for the third integral:

$$
f_{B_{\rho}^{+}(y)} I I I d x \leq L K(M) c(p, M)\left(\Phi^{2}(y, \rho, \xi)+\rho^{\alpha}\right) .
$$

Combining the estimates (26)-(28) in (25), the assertion follows in the particular case $\sup _{B_{\rho}^{+}(y)}|D \varphi| \leq 1$. Rescaling yields the general result.

The right hand side of the bound in Lemma 6.1 must be small in order to apply the $\mathcal{A}$-harmonic approximation, Lemma 4.4, to the function $w=u-\xi x_{n}$. Combined with the a priori estimates for $\mathcal{A}$-harmonic functions (i. e., solutions of a linear elliptic system with coefficients $\mathcal{A}$ ) this provides an estimate for the excess function on smaller half-balls. We proceed in a manner close to [20], section 3.3-3.4.

For a solution $u \in W_{\Gamma}^{1, p}\left(B^{+}, \mathbb{R}^{N}\right)$ of system $(24)$ we fix $y \in \Gamma, 0<\rho<1-|y|, M_{1} \geq 1, \xi \in \mathbb{R}^{N}$ with $|\xi| \leq M_{1}$ and we set

$$
\begin{aligned}
& \Phi(r, \xi):=\Phi(y, r, \xi)=\left(f_{B_{r}^{+}(y)}\left|V\left(D u-\xi \otimes e_{n}\right)\right|^{2} d x\right)^{\frac{1}{2}}, \\
& w:=u-\xi x_{n} \quad \in W_{\Gamma}^{1, p}\left(B^{+}, \mathbb{R}^{N}\right) .
\end{aligned}
$$

The bilinear form $\mathcal{A}:=\frac{1}{\nu} D_{z} A\left(y, 0, \xi \otimes e_{n}\right)$ is elliptic with ellipticity constant $\left(1+M_{1}^{2}\right)^{\frac{p-2}{2}}$ and bounded from above by $\frac{L}{\nu}$, see conditions $(\mathrm{H} 2)$ and (H3). Applying Lemma 6.1 with some free parameter $\delta \in(0,1]$ we calculate for all $\varphi \in C_{0}^{\infty}\left(B_{\rho}^{+}(y), \mathbb{R}^{N}\right)$ :

$$
\begin{aligned}
& \left|f_{B_{\rho}^{+}(y)} \mathcal{A}(D w, D \varphi) d x\right| \leq c_{a}\left(p, \frac{\nu}{L}, M_{1}, K\left(M_{1}\right)\right)\left[\Phi^{2}(\rho, \xi)+\rho^{\alpha}+\chi_{M_{1}}\left(\Phi^{2}(\rho, \xi)\right) \Phi(\rho, \xi)\right] \sup _{B_{\rho}^{+}(y)}|D \varphi| \\
& \leq c_{a} \sqrt{\Phi^{2}(\rho, \xi)+\delta^{-2} \rho^{2 \alpha}}\left[\Phi(\rho, \xi)+\delta+\chi_{M_{1}}\left(\Phi^{2}(\rho, \xi)\right)\right] \sup _{B_{\rho}^{+}(y)}|D \varphi| \\
& \leq 2 c_{a} \sqrt{\Phi^{2}(\rho, \xi)+\delta^{-2} \rho^{2 \alpha}} \sqrt{\Phi^{2}(\rho, \xi)+\frac{1}{2} \delta^{2}+\chi_{M_{1}}^{2}\left(\Phi^{2}(\rho, \xi)\right)} \sup _{B_{\rho}^{+}(y)}|D \varphi|
\end{aligned}
$$

Let $\varepsilon>0$ to be specified later, and let $\delta=\delta\left(n, N, p, \frac{\nu}{L}\left(1+M_{1}^{2}\right)^{\frac{p-2}{2}}, \varepsilon\right) \in(0,1]$ denote the constant from Lemma 4.4. Assume

$$
\begin{aligned}
& \Phi^{2}(\rho, \xi)+\chi_{M_{1}}^{2}\left(\Phi^{2}(\rho, \xi)\right) \leq \frac{1}{2} \delta^{2} \\
& \gamma:=2 c_{a} \sqrt{\Phi^{2}(\rho, \xi)+\delta^{-2} \rho^{2 \alpha}} \leq 1
\end{aligned}
$$

then we have due to $(29)$ the estimate $\left|f_{B_{\rho}^{+}(y)} \mathcal{A}(D w, D \varphi) d x\right| \leq \gamma \delta \sup _{B_{\rho}^{+}(y)}|D \varphi|$. Furthermore, the definition of $\Phi(\rho, \xi)$ yields $\left(c_{a} \geq 1\right)$ :

$$
f_{B_{\rho}^{+}(y)}|W(D w)|^{2} d x \leq f_{B_{\rho}^{+}(y)}|V(D w)|^{2} d x=\Phi^{2}(\rho, \xi) \leq \gamma^{2} .
$$


Under the smallness assumptions (30) and (31) we now find according to Lemma 4.4 the existence of a $\mathcal{A}$-harmonic function $h \in W_{\Gamma}^{1, p}\left(B_{\frac{\rho}{2}}^{+}(y), \mathbb{R}^{N}\right)$ such that

$$
f_{B_{\rho / 2}^{+}(y)}\left|W\left(\frac{w-\gamma h}{\rho}\right)\right|^{2} d x \leq \gamma^{2} \varepsilon \quad \text { and } \quad f_{B_{\rho / 2}^{+}(y)}|W(D h)|^{2} d x \leq 2^{n+2} .
$$

Now we deduce some relevant properties of the function $h$ : splitting the integration domain in $\{|D h|>1\}$ and $\{|D h| \leq 1\}$, we infer using Lemma 3.1(i), Hölder's inequality and (32)

$$
f_{B_{\rho / 2}^{+}(y)}|D h|^{p} d x \leq \sqrt{2}\left(f_{B_{\rho / 2}^{+}(y)}|W(D h)|^{2} d x+\left(f_{B_{\rho / 2}^{+}(y)}|W(D h)|^{2} d x\right)^{\frac{1}{2}}\right) \leq 2^{n+4} .
$$

Using Lemma 4.2, from which follows that $h$ is smooth on $\overline{B_{\bar{\rho}}^{+}(y)}$ for all $\bar{\rho}<\frac{\rho}{2}$, and the last estimate, we observe

$$
\sup _{B_{\rho / 4}^{+}(y)}\left(|D h|^{2}+\rho^{2}\left|D^{2} h\right|^{2}\right) \leq c\left(f_{B_{\rho / 2}^{+}(y)}\left|D_{n} h\right|^{p} d x\right)^{\frac{2}{p}} \leq c_{h}^{2}\left(n, N, p, \frac{\nu}{L}\left(1+M_{1}^{2}\right)^{\frac{p-2}{2}}\right) .
$$

Since $h$ vanishes on $\Gamma_{\frac{\rho}{2}}$ there exists a constant vector $\zeta \in \mathbb{R}^{N}$ such that we have the representation

$$
D h(y)=\zeta \otimes e_{n} \quad \text { where } \quad|\zeta| \leq c_{h} .
$$

For $x \in B_{2 \theta \rho}^{+}(y)$ (the parameter $\theta \in\left(0, \frac{1}{8}\right]$ will be fixed later), Taylor-expansion gives $h(x)=h(y)+$ $D h(y)(x-y)+R(x)=\zeta x_{n}+R(x)$ where the remainder term $R(x)$ is estimated via (33) by $|R(x)| \leq$ $\frac{1}{2} \sup _{B_{2 \theta \rho}^{+}(y)}\left|D^{2} h\right|(2 \theta \rho)^{2} \leq 2 c_{h} \theta^{2} \rho$. Thus we obtain

$$
\sup _{B_{2 \theta \rho}^{+}(y)}\left|h(x)-\zeta x_{n}\right|^{2} \leq \sup _{B_{2 \theta \rho}^{+}(y)}|R(x)|^{2} \leq 4 c_{h}^{2} \theta^{4} \rho^{2} .
$$

Now, using Lemma 3.1 (iii), (i) and (32), this implies:

$$
\begin{aligned}
& f_{B_{2 \theta \rho}^{+}(y)}\left|V\left(\frac{w-\gamma \zeta x_{n}}{2 \theta \rho}\right)\right|^{2} d x \leq c(p)\left(f_{B_{2 \theta \rho}^{+}(y)}\left|W\left(\frac{w-\gamma h}{2 \theta \rho}\right)\right|^{2} d x+f_{B_{2 \theta \rho}^{+}(y)}\left|\gamma \frac{h-\zeta x_{n}}{2 \theta \rho}\right|^{2} d x\right) \\
& \leq c(p)\left((2 \theta)^{-2}(4 \theta)^{-n} f_{B_{\rho / 2}^{+}(y)}\left|W\left(\frac{w-\gamma h}{\rho}\right)\right|^{2} d x+\gamma^{2}(2 \theta \rho)^{-2} \sup _{B_{2 \theta \rho}^{+}(y)}\left|h(x)-\zeta x_{n}\right|^{2}\right) \\
& \leq c(p)\left(\theta^{-n-2} \varepsilon+c_{h}^{2} \theta^{2}\right) \gamma^{2} \leq c(p) c_{h}^{2} \theta^{2} \gamma^{2},
\end{aligned}
$$

where we have chosen $\varepsilon=\theta^{n+4}$ in the last inequality. This fixes $\delta=\delta\left(n, N, p, \frac{\nu}{L}\left(1+M_{1}^{2}\right)^{\frac{p-2}{2}}, \varepsilon\right)$ in terms of $\theta$; without loss of generality, we may assume $\delta \in(0,1]$. In the next step we want to estimate the left-hand side of (35) by means of the Caccioppoli inequality. Since $w-\gamma \zeta x_{n}=u-(\xi+\gamma \zeta) x_{n}$ this is only possible if $|\xi+\gamma \zeta|$ is bounded. Thus we choose $M_{2} \geq M_{1}+1$ such that $|\xi+\gamma \zeta| \leq M_{2}$. Considering that the constants $c_{h}, \delta$ and $c_{a}$ depend monotone nondecreasingly on $M_{1}$ we note that it is sufficient to note the dependency on $M_{2}$.

Applying the Caccioppoli inequality from Lemma 5.1, we infer with (35) an estimate for the excess function on smaller half-balls $B_{\theta \rho}^{+}(y)$ :

$$
\begin{aligned}
\Phi^{2}(\theta \rho, \xi+\gamma \zeta) & =f_{B_{\theta \rho}^{+}(y)}\left|V\left(D u-(\xi+\gamma \zeta) \otimes e_{n}\right)\right|^{2} d x \\
& \leq c_{c a c c}\left(f_{B_{2 \theta}^{+}(y)}\left|V\left(\frac{w-\gamma \zeta x_{n}}{2 \theta \rho}\right)\right|^{2} d x+(2 \theta \rho)^{2 \alpha}\right) \\
& \leq c_{c a c c}\left(c(p) c_{h}^{2} \theta^{2} \gamma^{2}+\rho^{2 \alpha}\right) \leq c_{d e c}^{2}\left(\theta^{2} \Phi^{2}(\rho, \xi)+\delta^{-2} \rho^{2 \alpha}\right),
\end{aligned}
$$

where we have used the definition of $\gamma$ in the last line and where the constant $c_{d e c}=2 \sqrt{c_{c a c c} c(p)} c_{h} c_{a}$ depends only on $n, N, p, \frac{\nu}{L}, M_{2}$ and $K\left(M_{2}\right)$. To an arbitrary exponent $\sigma \in(\alpha, 1)$ we next fix $\theta=$ $\theta\left(n, N, p, \frac{\nu}{L}, M_{2}, K\left(M_{2}\right), \sigma\right) \leq \frac{1}{8}$ sufficiently small such that $c_{d e c}^{2} \theta^{2} \leq \theta^{2 \sigma}$ is satisfied. Note that this fixes 
$\delta$. With the definition $\widetilde{\Phi}^{2}(\rho, \xi):=\Phi^{2}(\rho, \xi)+\rho^{2 \alpha}$ of the modified excess function we come to the conclusion that

$$
\widetilde{\Phi}^{2}(\theta \rho, \xi+\gamma \zeta) \leq \theta^{2 \sigma} \widetilde{\Phi}^{2}(\rho, \xi)+\widetilde{c}_{d e c}^{2} \delta^{-2} \rho^{2 \alpha}
$$

where $\widetilde{c}_{d e c}^{2}=1+c_{d e c}^{2}$. If we now assume the smallness condition

$$
\widetilde{\Phi}^{2}(\rho, \xi)+\chi_{M_{2}}^{2}\left(\widetilde{\Phi}^{2}(\rho, \xi)\right) \leq \frac{\delta^{2}}{4 c_{a}^{2} c_{h}^{2}},
$$

we easily compute that the previous assumptions (30), (31) are satisfied (note that $\chi_{M}(t)$ is monotone in $M$ and $t$ ), and furthermore, we have $|\xi+\gamma \zeta| \leq M_{1}+1 \leq M_{2}$. Thus we have shown an excess improvement:

Lemma 6.2: Let $y \in \Gamma, M_{2}=2 M_{1} \geq 2$ and $\sigma \in(\alpha, 1)$ be fixed. Then there exists $\theta \in\left(0, \frac{1}{8}\right]$ and $\delta \in(0,1]$ depending on $n, N, p, \frac{\nu}{L}, M_{2}, K\left(M_{2}\right)$ and $\sigma$, such that the following holds: if for some $\xi \in \mathbb{R}^{N}$ with $|\xi| \leq M_{1}$ and a radius $\rho, 0<\rho<1-|y|$, the smallness assumption (36) is fulfilled, then there exists $a \zeta \in \mathbb{R}^{N}$ with $|\zeta| \leq c_{h}$ such that:

$$
\widetilde{\Phi}^{2}(\theta \rho, \xi+\gamma \zeta) \leq \theta^{2 \sigma} \widetilde{\Phi}^{2}(\rho, \xi)+\widetilde{c}_{d e c}^{2} \delta^{-2} \rho^{2 \alpha}
$$

with a constant $\widetilde{c}_{d e c}=\widetilde{c}_{d e c}\left(n, N, p, \frac{\nu}{L}, M_{2}, K\left(M_{2}\right)\right)$.

\section{Iteration for the model situation}

In the next step we show that the previous excess improvement can be iterated and that the quantities appearing are uniformly bounded. For $j \in \mathbb{N}_{0}$ we define inductively $\xi_{j}, \zeta_{j} \in \mathbb{R}^{N}, \gamma_{j} \in \mathbb{R}$ and functions $w_{j} \in W_{\Gamma}^{1, p}\left(B_{\theta^{j} \rho}^{+}(y), \mathbb{R}^{N}\right), h_{j} \in W_{\Gamma}^{1, p}\left(B_{\theta^{j} \frac{\rho}{2}}^{+}(y), \mathbb{R}^{N}\right)$ by:

$$
\xi_{j}:=\xi+\sum_{k=0}^{j-1} \gamma_{k} \zeta_{k}, \quad \gamma_{j}:=2 c_{a} \sqrt{\Phi^{2}\left(\theta^{j} \rho, \xi_{j}\right)+\delta^{-2}\left(\theta^{j} \rho\right)^{2 \alpha}}, \quad w_{j}:=u-\xi_{j} x_{n}
$$

(i. e., the quantities with indices 0 correspond to the original ones). For the definition of $\zeta_{j}$ and $h_{j}$ respectively, we have to proceed analogously to the proof of the last lemma: as long as the smallness conditions

$$
\left|\xi_{j}\right| \leq M_{2} \quad \text { and } \quad \widetilde{\Phi}^{2}\left(\theta^{j} \rho, \xi_{j}\right)+\chi_{M_{2}}^{2}\left(\widetilde{\Phi}^{2}\left(\theta^{j} \rho, \xi_{j}\right)\right) \leq \frac{\delta^{2}}{4 c_{a}^{2} c_{h}^{2}}
$$

are satisfied for $j \in \mathbb{N}$, the assumptions of Lemma 4.4 are fulfilled. Thus we obtain the existence of $\frac{1}{\nu} D_{z} A\left(y, 0, \xi_{j} \otimes e_{n}\right)$-harmonic functions $h_{j} \in W_{\Gamma}^{1, p}\left(B_{\theta^{j} \frac{\rho}{2}}^{+}(y), \mathbb{R}^{N}\right)$ for which the corresponding estimates (32) hold true:

$$
f_{B_{\theta^{j} \frac{\rho}{2}}^{+}(y)}\left|W\left(\frac{w_{j}-\gamma_{j} h_{j}}{\theta^{j} \rho}\right)\right|^{2} d x \leq \gamma_{j}^{2} \varepsilon, \quad f_{B_{\theta^{j} \frac{\rho}{2}}^{+}(y)}\left|W\left(D h_{j}\right)\right|^{2} d x \leq 2^{n+2}
$$

Next we define $\zeta_{j}$ via $D h_{j}(y)=\zeta_{j} \otimes e_{n}$; in the next lemma we will show $\left|\zeta_{j}\right| \leq c_{h}$. Given this if $\left|\xi_{j+1}\right| \leq M_{2}$, we can use the smallness conditions in (37) and calculate analogously to above to obtain

$$
\widetilde{\Phi}^{2}\left(\theta^{j+1} \rho, \xi_{j+1}\right) \leq \theta^{2 \sigma} \widetilde{\Phi}^{2}\left(\theta^{j} \rho, \xi_{j}\right)+\widetilde{c}_{d e c}^{2} \delta^{-2}\left(\theta^{j} \rho\right)^{2 \alpha}
$$

For fixed $M_{2}>0$ we choose $t_{0}>0$ such that

$$
t_{0}^{2}+\chi_{M_{2}}^{2}\left(t_{0}^{2}\right) \leq \frac{\delta^{2}}{4 c_{a}^{2} c_{h}^{2}} \quad \text { and } \quad t_{0} \leq \frac{M_{2}\left(1-\theta^{\alpha}\right)}{8 c_{a} c_{h}} .
$$

Now we choose a radius $\rho_{0} \in(0,1-|y|)$ satisfying

$$
\frac{2 \widetilde{c}_{d e c}^{2} \delta^{-2}}{\theta^{2 \alpha}-\theta^{2 \sigma}} \rho_{0}^{2 \alpha} \leq t_{0}^{2}
$$

Therefore, $t_{0}$ and $\rho_{0}$ depend only on $n, N, p, \frac{\nu}{L}, M_{2}, K\left(M_{2}\right), \alpha, \sigma$ and $\chi_{M_{2}}(\cdot)$. 
Lemma 6.3 (cf. [20], Proposition 3.5): Let $M_{2} \geq 2$. Choose $t_{0}$ and $\rho_{0}$ such that (40) and (41) are valid. Assume that for some $\rho \in\left(0, \rho_{0}\right]$ we have:

$$
\left|\xi_{0}\right| \leq \frac{1}{2} M_{2} \quad \text { and } \quad \widetilde{\Phi}^{2}\left(\rho, \xi_{0}\right) \leq \frac{1}{2} t_{0}^{2} .
$$

Then for all $j \in \mathbb{N}_{0}$ we have the following estimates:

$$
\begin{aligned}
\left|\zeta_{j}\right| & \leq c_{h} \\
\widetilde{\Phi}\left(\theta^{j} \rho, \xi_{j}\right) & \leq \theta^{\alpha j} t_{0} \\
\gamma_{j} & \leq 4 c_{a} \theta^{\alpha j} t_{0} \\
\left|\xi_{j}\right| & \leq M_{2} .
\end{aligned}
$$

Furthermore, the limit $\xi_{\infty}:=\lim _{j \rightarrow \infty} \xi_{j}$ exists and for any $r \in(0, \rho]$ there holds

$$
f_{B_{r}^{+}(y)}\left|V\left(D u-\xi_{\infty} \otimes e_{n}\right)\right|^{2} d x \leq c_{i t}\left(\left(\frac{r}{\rho}\right)^{2 \sigma} \Phi^{2}\left(\rho, \xi_{0}\right)+r^{2 \alpha}\right)
$$

for a constant $c_{i t}=c\left(n, N, p, \frac{\nu}{L}, M_{2}, K\left(M_{2}\right), \alpha, \sigma, \chi_{M_{2}}(\cdot)\right)$.

Proof: Analogously to (34) we use $(38)_{j}$ to derive (i) ${ }_{j}$ for any $j \in \mathbb{N}_{0}$. We shall establish (ii)-(iv) by induction. We first note that (ii) $)_{0}$ and (iv) $)_{0}$ follow immediately from (42), and for (iii) $)_{0}$ we additionally use the assumption (41) on the radius to see:

$$
\gamma_{0}^{2}=4 c_{a}^{2}\left(\Phi^{2}\left(\rho, \xi_{0}\right)+\delta^{-2} \rho^{2 \alpha}\right) \leq 4 c_{a}^{2} t_{0}^{2} .
$$

Now we assume that for a given $j \in \mathbb{N}$ the estimates (ii) $l_{l^{-}}$(iv) $\ell$ are valid for $\ell \in\{0, \ldots, j-1\}$. Now (iv) $j$ follows using (iii), (i), (42) and (40):

$$
\left|\xi_{j}\right| \leq\left|\xi_{0}\right|+\sum_{\ell=0}^{j-1} \gamma_{\ell}\left|\zeta_{\ell}\right| \leq \frac{1}{2} M_{2}+4 c_{a} c_{h} t_{0} \sum_{\ell=0}^{j-1} \theta^{\alpha \ell} \leq \frac{1}{2} M_{2}+\frac{1}{2} \frac{8 c_{a} c_{h} t_{0}}{1-\theta^{\alpha}} \leq M_{2} .
$$

In particular, the first condition in $(37)_{\ell}$ is satisfied due to (iv) $\ell$, and the second condition due to (ii) $\ell$ and (40). Since also (iv) ${ }_{j}$ is valid, the estimate $(39)_{\ell}$ holds true for $\ell<j$. Applying in turn $(39)_{j-1}, \ldots,(39)_{0}$, we estimate:

$$
\begin{aligned}
\widetilde{\Phi}^{2}\left(\theta^{j} \rho, \xi_{j}\right) & \leq \theta^{2 \sigma} \widetilde{\Phi}^{2}\left(\theta^{j-1} \rho, \xi_{j-1}\right)+\widetilde{c}_{d e c}^{2} \delta^{-2}\left(\theta^{j-1} \rho\right)^{2 \alpha} \\
& \leq \theta^{2 \sigma j} \widetilde{\Phi}^{2}\left(\rho, \xi_{0}\right)+\frac{\widetilde{c}_{d e c}^{2} \delta^{-2}}{\theta^{2 \alpha}-\theta^{2 \sigma}}\left(\theta^{j} \rho\right)^{2 \alpha} .
\end{aligned}
$$

Therefore, the fact that $\theta^{2 \sigma} \leq \theta^{2 \alpha}$, the assumption (42) and the choice of $\rho_{0}$ in (41) imply the estimate (ii) ${ }_{j}$ :

$$
\widetilde{\Phi}^{2}\left(\theta^{j} \rho, \xi_{j}\right) \leq \theta^{2 \alpha j}\left(\widetilde{\Phi}^{2}\left(\rho, \xi_{0}\right)+\frac{\widetilde{c}_{d e c}^{2} \delta^{-2}}{\theta^{2 \alpha}-\theta^{2 \sigma}} \rho_{0}^{2 \alpha}\right) \leq \theta^{2 \alpha j} t_{0}^{2}
$$

Using the definition of $\gamma_{j}$ we compute from (44)

$$
\begin{aligned}
\left|\gamma_{j}\right|^{2} & \leq 4 c_{a}^{2}\left(\theta^{2 \sigma j} \widetilde{\Phi}^{2}\left(\rho, \xi_{0}\right)+\frac{\widetilde{c}_{d e c}^{2} \delta^{-2}}{\theta^{2 \alpha}-\theta^{2 \sigma}}\left(\theta^{j} \rho\right)^{2 \alpha}+\delta^{-2}\left(\theta^{j} \rho\right)^{2 \alpha}\right) \\
& \leq 4 c_{a}^{2}\left(\theta^{2 \sigma j} \widetilde{\Phi}^{2}\left(\rho, \xi_{0}\right)+\frac{2 \widetilde{c}_{d e c}^{2} \delta^{-2}}{\theta^{2 \alpha}-\theta^{2 \sigma}}\left(\theta^{j} \rho\right)^{2 \alpha}\right) .
\end{aligned}
$$

Thus (iii) ${ }_{j}$ is valid due to (41) and (42).

In what follows we abbreviate $c_{1}^{2}:=\frac{\widetilde{\tau}_{d e c}^{2} \delta^{-2}}{\theta^{2 \alpha}-\theta^{2 \sigma}}$. We next show the existence of the limit of $\left\{\xi_{j}\right\}$. For $i>j$, (i) and (45) allow us to estimate

$$
\left|\xi_{i}-\xi_{j}\right|=\left|\sum_{\ell=j}^{i-1} \gamma_{\ell} \zeta_{\ell}\right| \leq 2 c_{h} c_{a}\left(\widetilde{\Phi}\left(\rho, \xi_{0}\right) \sum_{\ell=j}^{i-1} \theta^{\sigma \ell}+\sqrt{2} c_{1} \rho^{\alpha} \sum_{\ell=j}^{i-1} \theta^{\alpha \ell}\right) \leq c_{2}\left(\theta^{2 \sigma j} \widetilde{\Phi}^{2}\left(\rho, \xi_{0}\right)+\left(\theta^{j} \rho\right)^{2 \alpha}\right)^{\frac{1}{2}}
$$


where $c_{2}=4 c_{h} c_{a}\left(1+c_{1}\right)\left(1-\theta^{\alpha}\right)^{-1}$. The right-hand side approaches 0 as $j \rightarrow \infty$; thus $\left\{\xi_{j}\right\}$ is a Cauchy sequence and converges to some $\xi_{\infty} \in \mathbb{R}^{N}$. Letting $i$ tend to infinity we have:

$$
\left|\xi_{\infty}-\xi_{j}\right| \leq c_{2}\left(\theta^{2 \sigma j} \Phi^{2}\left(\rho, \xi_{0}\right)+\left(\theta^{j} \rho\right)^{2 \alpha}\right)^{\frac{1}{2}}
$$

As a consequence, we note via 3.1 (iii), (i) and (44):

$$
f_{B_{\theta^{j} \rho}^{+}(y)}\left|V\left(D u-\xi_{\infty} \otimes e_{n}\right)\right|^{2} d x \leq c(p)\left(\widetilde{\Phi}^{2}\left(\theta^{j} \rho, \xi_{j}\right)+\left|\xi_{\infty}-\xi_{j}\right|^{2}\right) \leq c_{3}\left(\theta^{2 \sigma j} \widetilde{\Phi}^{2}\left(\rho, \xi_{0}\right)+\left(\theta^{j} \rho\right)^{2 \alpha}\right)
$$

for $c_{3}=c(p)\left(c_{1}^{2}+c_{2}^{2}\right)$. For a continuous version of the last inequality we consider an arbitrary radius $r \in(0, \rho]$ and use the fact that there exists a unique $k \in \mathbb{N}_{0}$ such that $\theta^{k+1} \rho<r \leq \theta^{k} \rho$. This finally gives the desired result with $c_{i t}=2 \theta^{-n-2 \sigma} c_{3}$.

Excess decay estimate in the interior: The analogous excess-decay estimate in the interior is established in a similar way: for $B_{\rho}\left(x_{0}\right) \subset \subset \Omega$ we freeze our system in $\mathcal{A}=D_{z} A\left(x_{0},(u)_{x_{0}, \rho}, \Upsilon\right)$ for some $\Upsilon \in \mathbb{R}^{n N}$ where $\left|(u)_{x_{0}, \rho}\right|,|\Upsilon| \leq M$. Defining the excess function in $x_{0}$ for the interior by

$$
\Psi\left(x_{0}, \rho, \Upsilon\right):=\left(f_{B_{\rho}\left(x_{0}\right)}|V(D u-\Upsilon)|^{2} d x\right)^{\frac{1}{2}},
$$

$\widetilde{\Psi}^{2}\left(x_{0}, \rho, \Upsilon\right):=\Psi^{2}\left(x_{0}, \rho, \Upsilon\right)+\rho^{2 \alpha}$, we find via approximate $\mathcal{A}$-harmonicity and an iteration technique: let $\widehat{M}_{2} \geq 2$ and let $t_{1}$ be sufficiently small depending on $n, N, p, \frac{\nu}{L}, \widehat{M}_{2}, K\left(\widehat{M}_{2}\right), \alpha, \sigma$ and $\chi_{\widehat{M}_{2}}$ such that

$$
t_{1}^{2}+\chi_{\widehat{M}_{2}}^{2}\left(t_{1}^{2}\right) \leq \frac{\widehat{\delta}^{2}}{4 \widehat{c}_{i}^{2} \widehat{c}_{a}^{2} \widehat{c}_{h}^{2}} \quad \text { and } \quad t_{1} \leq \frac{\widehat{M}_{2}\left(1-\widehat{\theta}^{\alpha}\right)(2 \widehat{\theta})^{n}}{8 c_{i} \widehat{c}_{a} \widehat{c}_{h}}
$$

(where the quantities signed with ${ }^{\wedge}$ are the quantities corresponding to the boundary situation, and $c_{i}$ depends on $n$ and $p$ ). Choose the radius $\rho_{1}$ with the same dependencies such that

$$
\frac{2 \widehat{c}_{d e c}^{2} \widehat{\delta}^{-2}}{\widehat{\theta}^{2 \alpha}-\widehat{\theta}^{2 \sigma}} \rho_{1}^{2 \alpha} \leq t_{1}^{2} .
$$

If, for some $\rho \in\left(0, \rho_{1}\right]$, we have

$$
\left|\Upsilon_{0}\right| \leq \frac{1}{2} \widehat{M}_{2}, \quad\left|(u)_{x_{0}, \rho}\right| \leq \frac{1}{2} \widehat{M}_{2} \quad \text { and } \quad \widetilde{\Psi}^{2}\left(x_{0}, \rho, \Upsilon_{0}\right) \leq \frac{1}{2} t_{1}^{2}
$$

then there exists an $\Upsilon_{\infty} \in \mathbb{R}^{n N}$ such that for any $r \in(0, \rho]$ we have

$$
f_{B_{r}\left(x_{0}\right)}\left|V(D u)-V\left(\Upsilon_{\infty}\right)\right|^{2} d x \leq \widehat{c}_{i t}\left(\left(\frac{r}{\rho}\right)^{2 \sigma} \Psi^{2}\left(x_{0}, \rho, \Upsilon_{0}\right)+r^{2 \alpha}\right) .
$$

We want to remark here that in the interior situation weak solutions are not a priori bounded. Therefore, we further need to iterate the averages of $u$ and show that the sequence $\left|(u)_{x_{0}, \widehat{\theta}^{j} \rho}\right|$ is bounded. This is possible in a standard way using the Poincaré inequality and estimates involving the excess function (cf. e. g. [10], estimate (3.39) or see [2], proof of Lemma 6.6).

\section{Proof of the main results}

We next want to prove the main theorems stated in section 2. For this purpose we show that the assumptions for the excess-decay estimate in Lemma 6.3 for the boundary case and the analogous estimate in the interior are satisfied in the set of regular points.

Proof (of Theorem 2.1): Consider $x_{0} \in \Omega \backslash\left(\Pi_{1} \cup \Pi_{2}\right)$. Then there exist $\widehat{M}_{2} \geq 2$ and $0<\rho \leq \rho_{1}$ such that: $B_{2 \rho}\left(x_{0}\right) \subset \subset \Omega$ and

$$
\begin{aligned}
& \left|(u)_{x_{0}, \rho}\right|<\frac{1}{2} \widehat{M}_{2}, \quad\left|(V(D u))_{x_{0}, \rho}\right|<2^{\frac{p-6}{4}} \widehat{M}_{2}^{\frac{p}{2}} \text { and } \\
& f_{B_{\rho}\left(x_{0}\right)}\left|V(D u)-(V(D u))_{x_{0}, \rho}\right|^{2} d x+\rho^{2 \alpha}<\frac{1}{2} c^{-2}\left(p, \widehat{M}_{2}\right) t_{1}^{2}
\end{aligned}
$$


where $c\left(p, \widehat{M}_{2}\right)$ is coming from Lemma 3.1 (vi). Since the functions

$$
z \mapsto(u)_{z, \rho}, \quad z \mapsto(V(D u))_{z, \rho} \quad \text { and } \quad z \mapsto f_{B_{\rho}(z)}\left|V(D u)-(V(D u))_{z, \rho}\right|^{2} d x
$$

are continuous, there exists a ball $B_{\tilde{\rho}}\left(x_{0}\right)$ such that for all points $z \in B_{\tilde{\rho}}\left(x_{0}\right)$ we have: $B_{\rho}(z) \subset \subset \Omega$ and the estimates (50) and (51) hold with $x_{0}$ replaced by $z$.

We now choose $\Upsilon_{0}(z) \in \mathbb{R}^{n N}$ such that $V\left(\Upsilon_{0}(z)\right)=(V(D u))_{z, \rho}$ (note: this is always possible as the function $V$ is bijective). Combining these estimates with Lemma 3.1 (i) and (vi) we find that $\left|\Upsilon_{0}(z)\right|<$ $\frac{1}{2} \widehat{M}_{2}$ and $\widetilde{\Psi}^{2}\left(z, \rho, \Upsilon_{0}(z)\right)<\frac{1}{2} t_{1}^{2}$. Thus the above assumptions in (48) are satisfied for all $z \in B_{\tilde{\rho}}\left(x_{0}\right)$ and we obtain: there exists $\Upsilon_{\infty}(z) \in \mathbb{R}^{n N}$ such that:

$$
f_{B_{r}(z)}\left|V(D u)-V\left(\Upsilon_{\infty}(z)\right)\right|^{2} d x \leq c\left(\left(\frac{r}{\rho}\right)^{2 \sigma} \Psi^{2}\left(z, \rho, \Upsilon_{0}(z)\right)+r^{2 \alpha}\right)
$$

for any radius $0<r \leq \rho$ and all $z \in B_{\tilde{\rho}}\left(x_{0}\right)$. The constant $c$ depends on $n, N, p, \frac{\nu}{L}, \widehat{M}_{2}, K\left(2 \widehat{M}_{2}\right), \alpha, \sigma$ and $\chi_{\widehat{M}_{2}}(\cdot)$. Applying the integral characterization of Hölder-continuous functions due to Campanato (see [26], chapter 1.1, Lemma 1) we conclude that there exists a representative of $V \circ D u$ which is Höldercontinuous with exponent $\alpha(<\sigma)$. Using Lemma 3.2 and as well as Lebesgue's Differentiation Theorem we obtain the desired result.

For the proof of the characterization of regular boundary points in Theorem 2.2 we first consider the set of regular points $\operatorname{Reg}_{\Gamma} u$ defined corresponding to the definition of $\operatorname{Reg}_{\partial \Omega} u$ in the model situation. Here we make use of a slight modification of Campanato's integral characterization of Hölder-continuity:

Theorem 7.1 ([20], Theorem 2.3): Consider $n \in \mathbb{N}, n \geq 2$ and $x_{0} \in \mathbb{R}^{n-1} \times\{0\}$. Suppose that there are positive constants $\alpha \in(0,1], \kappa>0$ such that, for some $v \in L^{2}\left(B_{6 R}^{+}\left(x_{0}\right)\right)$, there holds the following:

$$
\inf _{\mu \in \mathbb{R}}\left\{f_{B_{\rho}^{+}(y)}|v-\mu|^{2} d x\right\} \leq \kappa^{2}\left(\frac{\rho}{R}\right)^{2 \alpha}
$$

for all $y \in \Gamma_{2 R}\left(x_{0}\right)$ and $\rho \leq 4 R ;$ and

$$
\inf _{\mu \in \mathbb{R}}\left\{f_{B_{\rho}(y)}|v-\mu|^{2} d x\right\} \leq \kappa^{2}\left(\frac{\rho}{R}\right)^{2 \alpha}
$$

for all $y \in B_{2 R}^{+}\left(x_{0}\right)$ mit $B_{\rho}(y) \subset B_{2 R}^{+}\left(x_{0}\right)$. Then there exists a Hölder-continuous representative $\bar{v}$ of $v$ on $\overline{B_{R}^{+}}\left(x_{0}\right)$, and for $\bar{v}$ there holds: $|\bar{v}(x)-\bar{v}(z)| \leq c \kappa\left(\frac{|x-z|}{R}\right)^{\alpha}$ for all $x, z \in \overline{B_{R}^{+}}\left(x_{0}\right)$, for a constant $c$ depending only on $n$ and $\alpha$.

We then infer the desired charaterization in the model situation:

Theorem 7.2: Let $u \in W^{1, p}\left(B^{+}, \mathbb{R}^{N}\right)$ be a weak solution of $\operatorname{div} A(\cdot, u, D u)=0$ in $B^{+}$where the coefficients $A: B^{+} \times \mathbb{R}^{N} \times \mathbb{R}^{n N} \rightarrow \mathbb{R}^{n N}$ satisfy the assumptions (H1)-(H4). Then we have: for points $y \in \operatorname{Reg}_{\Gamma} u$ there holds that $D u$ is Hölder-continuous with exponent $\alpha$ in a neighbourhood of $y$ in $\overline{B^{+}}$, and $\operatorname{Sing}_{\Gamma} u \subset \Sigma_{1} \cup \Sigma_{2}$ where

$$
\begin{aligned}
& \Sigma_{1}=\left\{y \in \Gamma: \liminf _{\rho \rightarrow 0^{+}} f_{B_{\rho}^{+}(y)}\left|V\left(D_{n} u\right)-\left(V\left(D_{n} u\right)\right)_{y, \rho}^{+}\right|^{2} d x>0\right\}, \\
& \Sigma_{2}=\left\{y \in \Gamma: \limsup _{\rho \rightarrow 0^{+}}\left|\left(V\left(D_{n} u\right)\right)_{y, \rho}^{+}\right|=\infty\right\} .
\end{aligned}
$$

PRoOF: In the first step of the proof we will find a different formulation for the set $\Sigma_{1} \cup \Sigma_{2}$ which allows us to apply Lemma 6.3, where an assumption on the total weak derivative, instead of only the normal derivative of $u$, is required. Let $y \in \Gamma \backslash\left(\Sigma_{1} \cup \Sigma_{2}\right)$ and $\left\{\rho_{k}\right\}$ be a monotone decreasing sequence of radii with $\rho_{k} \rightarrow 0$ for $k \rightarrow \infty$ and

$$
\lim _{k \rightarrow \infty} f_{B_{\rho_{k}}^{+}(y)}\left|V\left(D_{n} u\right)-\left(V\left(D_{n} u\right)\right)_{y, \rho_{k}}^{+}\right|^{2} d x=0 .
$$


Since $y \notin \Sigma_{2}$ there exists $M \geq 1$ such that $\left|\left(V\left(D_{n} u\right)\right)_{y, \rho_{k}}^{+}\right| \leq M$ for all $k \in \mathbb{N}$. Similar to the proof of the interior estimate in Theorem 2.1 we define $\left\{\xi\left(y, \rho_{k}\right)\right\} \in \mathbb{R}^{N}$ via $V\left(\xi\left(y, \rho_{k}\right)\right)=\left(V\left(D_{n} u\right)\right)_{y, \rho_{k}}^{+}$. Then Lemma 3.1 (i) yields $\left|\xi\left(y, \rho_{k}\right)\right| \leq 2 M^{2}$. Applying the Caccioppoli inequality, the Poincaré inequality in Lemma 3.6 (note that $u-\xi\left(y, \rho_{k}\right) x_{n} \in W_{\Gamma}^{1, p}\left(B^{+}, \mathbb{R}^{N}\right)$ ) and Lemma 3.1 (vi) we compute

$$
\begin{aligned}
& f_{B_{\rho_{k} / 2}^{+}(y)}\left|V\left(D u-\xi\left(y, \rho_{k}\right) \otimes e_{n}\right)\right|^{2} d x \leq c_{c a c c}\left(f_{B_{\rho_{k}}^{+}(y)}\left|V\left(\frac{u-\xi\left(y, \rho_{k}\right) x_{n}}{\rho_{k}}\right)\right|^{2} d x+\rho_{k}^{2 \alpha}\right) \\
& \leq c_{c a c c}\left(c_{P}(p) f_{B_{\rho_{k}}^{+}(y)}\left|V\left(D_{n} u-\xi\left(y, \rho_{k}\right)\right)\right|^{2} d x+\rho_{k}^{2 \alpha}\right) \\
& \leq c\left(n, p, \frac{\nu}{L}, M, K\left(2 M^{2}\right)\right)\left(f_{B_{\rho_{k}}^{+}(y)}\left|V\left(D_{n} u\right)-\left(V\left(D_{n} u\right)\right)_{y, \rho_{k}}^{+}\right|^{2} d x+\rho_{k}^{2 \alpha}\right)
\end{aligned}
$$

which vanishes as $k \rightarrow \infty$. This calculation - performed in the case $p=2$ where the $V$-function acts as the identity function - shows that the characterization of regular boundary points given by Grotowski in [20] and by Hamburger in [22] are in fact equivalent as mentioned in the introduction. We now set

$$
\begin{aligned}
& \Sigma_{1}^{\prime}=\left\{y \in \Gamma: \liminf _{\rho \rightarrow 0^{+}} f_{B_{\rho}^{+}(y)}\left|V\left(D u-\xi(y, 2 \rho) \otimes e_{n}\right)\right|^{2} d x>0\right\} \\
& \Sigma_{2}^{\prime}=\left\{y \in \Gamma: \limsup _{\rho \rightarrow 0^{+}}|\xi(y, \rho)|=\infty\right\} .
\end{aligned}
$$

Then the calculation above yields the inclusion $\Gamma \backslash\left(\Sigma_{1} \cup \Sigma_{2}\right) \subset \Gamma \backslash\left(\Sigma_{1}^{\prime} \cup \Sigma_{2}^{\prime}\right)$ (moreover, using the fact that for any function $v \in L^{2}\left(\Omega, \mathbb{R}^{N}\right)$ the mean value minimizes the function $\mathbb{R}^{N} \ni \mu \mapsto \int_{\Omega}|v-\mu|^{2} d x$ combined with Lemma $3.1(\mathrm{v})$, we derive that the union of these sets are equivalent).

We thus consider $y \in \Gamma \backslash\left(\Sigma_{1} \cup \Sigma_{2}\right)$; without loss of generality, we may assume $y=0$. In the second step of the proof we will show: $D u \in C^{0, \alpha}\left(\overline{B_{\rho}^{+}}, \mathbb{R}^{n N}\right)$ for some $\rho>0$.

Let $M_{2}$ denote the upper bound on $2|\xi(0, \rho)|$ (note $M_{2}<\infty$ is guaranteed since $0 \notin \Sigma_{2}^{\prime}$ ). We take $t_{0}$ to be the constant according to Lemma 6.3 and let $\rho_{0}$ be the corresponding radius (cf. (40) and (41)). In order to apply Theorem 7.1 to conclude the Hölder-continuity up to the boundary, we have to combine the excess-decay estimates in the interior and at the boundary. Thus we define $\widehat{M}_{2}=2 M_{2}$ and choose $t_{1}$ according to the smallness assumption in (46). For any $y \in B^{+}$let $\rho_{1} \in\left(0, \min \left\{1-|y|, y_{n}\right\}\right)$ be the corresponding radius from (47). We now choose $t_{2}>0$ such that:

$$
t_{2}^{2} \leq \min \left\{t_{0}^{2}, 2^{1-n} 3^{-2 \sigma} c_{i t}^{-1} t_{1}^{2}\right\} \quad \text { and } \quad 2^{n+1} 3^{2 \sigma} c_{i t} t_{2} \leq M_{2}
$$

where $c_{i t}$ denotes the constant in Lemma 6.3. We fix a radius $R_{0}>0$ sufficiently small with

$$
6 R_{0} \leq \min \left\{\rho_{0}, \rho_{1}\right\} \quad \text { and } \quad 3^{n} 2^{3+4 \alpha} R_{0}^{2 \alpha} \leq t_{2}^{2}
$$

Since $0 \notin \Sigma_{1}^{\prime}$ we find some $R \in\left(0, R_{0}\right]$ such that, abbreviating $\xi_{0}(0):=\xi(0,12 R)$, we have:

$$
\Phi^{2}\left(0,6 R, \xi_{0}(0)\right)=f_{B_{6 R}^{+}(0)}\left|V\left(D u-\xi_{0}(0) \otimes e_{n}\right)\right|^{2} d x \leq 2^{-3} 3^{-n} t_{2}^{2}
$$

and by assumption $\left|\xi_{0}(0)\right| \leq \frac{1}{2} M_{2}$. Conditions (52) and (53) guarantee in particular that also the smallness assumption $\widetilde{\Phi}^{2}\left(0,6 R, \xi_{0}(0)\right) \leq \frac{1}{2} t_{0}^{2}$ of Lemma 6.3 is satisfied on $B_{6 R}^{+}(0)$. Thus we find $\xi_{\infty}(0) \in$ $\mathbb{R}^{N}$ such that for any $r \in(0,6 R]$ there holds:

$$
f_{B_{r}^{+}}\left|V\left(D u-\xi_{\infty}(0) \otimes e_{n}\right)\right|^{2} d x \leq c_{i t}\left[\left(\frac{r}{6 R}\right)^{2 \sigma} \Phi^{2}\left(0,6 R, \xi_{0}(0)\right)+r^{2 \alpha}\right] .
$$

Using the smallness assumption (54) we now want to show that the conditions of Theorem 7.1 are fulfilled on all required balls and half-balls with centre $y \in \Gamma_{2 R}$ and $y \in B_{2 R}^{+}$, respectively. We distinguish several cases (cf. [20], p. 378-379): 
Case 1: $y \in \Gamma_{2 R},|y| \leq \rho \leq 4 R$ :

Using $B_{\rho}^{+}(y) \subseteq B_{\rho+|y|}^{+}(0)$, the last estimate for $r=\rho+|y| \leq 6 R$ and $2^{2 \alpha} \leq 2^{2 \sigma}$, we see

$$
f_{B_{\rho}^{+}(y)}\left|V\left(D u-\xi_{\infty}(0) \otimes e_{n}\right)\right|^{2} d x \leq 2^{n+2 \sigma} c_{i t}\left[\left(\frac{\rho}{6 R}\right)^{2 \sigma} \Phi^{2}\left(0,6 R, \xi_{0}(0)\right)+\rho^{2 \alpha}\right] .
$$

Case 2: $y \in \Gamma_{2 R}, 0<\rho<|y|<2 R$ :

Here we calculate that the assumptions of Lemma 6.3 are also satisfied for the point $y$ and radius $2 R$ : recalling the definition of $\Phi\left(0,6 R, \xi_{0}(0)\right)$ we infer from (54) that:

$$
f_{B_{2 R}^{+}(y)}\left|V\left(D u-\xi_{0}(0) \otimes e_{n}\right)\right|^{2} d x \leq 3^{n} \Phi^{2}\left(0,6 R, \xi_{0}(0)\right) .
$$

We have $\left|\xi_{0}(0)\right| \leq \frac{1}{2} M_{2}$ (see above). Furthermore, by the condition (54) on $\Phi^{2}\left(0,6 R, \xi_{0}(0)\right)$ and (53) on the radius we conclude $\widetilde{\Phi}^{2}\left(y, 2 R, \xi_{0}(0)\right) \leq \frac{1}{2} t_{0}^{2}$. Lemma 6.3 now yields the existence of $\xi_{\infty}(y) \in \mathbb{R}^{N}$ with $\left|\xi_{\infty}(y)\right| \leq M_{2}$ such that for all $0<\rho \leq 2 R$ from (56) follows:

$$
f_{B_{\rho}^{+}(y)}\left|V\left(D u-\xi_{\infty}(y) \otimes e_{n}\right)\right|^{2} d x \leq 3^{n} c_{i t}\left[\left(\frac{\rho}{6 R}\right)^{2 \sigma} \Phi^{2}\left(0,6 R, \xi_{0}(0)\right)+\rho^{2 \alpha}\right] .
$$

Case 3: $y \in B_{2 R}^{+}, B_{\rho}(y) \subset B_{2 R}^{+}$:

Let $y^{\prime}=\left(y_{1}, \ldots, y_{n-1}, 0\right)$ be the projection of $y$ onto $\mathbb{R}^{n-1} \times\{0\}$. Here we have the inclusions

$$
B_{\rho}(y) \subset B_{y_{n}}(y) \subset B_{2 y_{n}}^{+}\left(y^{\prime}\right) .
$$

We shall now show that the assumptions for the iteration and thus for the excess-decay estimate in the interior are satisfied on the ball $B_{y_{n}}(y)$. If $\left|y^{\prime}\right| \leq 2 y_{n}(\leq 4 R)$ we can apply case 1 with centre $y^{\prime}$ and radius $2 y_{n}$ to obtain

$$
f_{B_{2 y_{n}}^{+}\left(y^{\prime}\right)}\left|V\left(D u-\hat{\xi} \otimes e_{n}\right)\right|^{2} d x \leq 3^{n+2 \sigma} c_{i t}\left[\left(\frac{2 y_{n}}{6 R}\right)^{2 \sigma} \Phi^{2}\left(0,6 R, \xi_{0}(0)\right)+\left(2 y_{n}\right)^{2 \alpha}\right] .
$$

Here we have set $\hat{\xi}=\xi_{\infty}(0)$ and have replaced $2^{n+2 \sigma}$ by $3^{n+2 \sigma}$. Otherwise if $2 y_{n}<\left|y^{\prime}\right|<2 R$ we have in particular $B_{2 y_{n}}^{+}\left(y^{\prime}\right) \subset B_{2 R}^{+}\left(y^{\prime}\right)$. This yields the existence of $\xi_{\infty}\left(y^{\prime}\right) \in \mathbb{R}^{N}$ with $\left|\xi_{\infty}\left(y^{\prime}\right)\right| \leq M_{2}$ such that the above inequality holds setting $\hat{\xi}=\xi_{\infty}\left(y^{\prime}\right)$.

Thus for any $y \in B_{2 R}^{+}$and $B_{\rho}(y) \subset B_{2 R}^{+}$we conclude, with the appropriate choice $\hat{\xi}=\xi_{\infty}(0)$ or $\hat{\xi}=\xi_{\infty}\left(y^{\prime}\right)$ that (keeping in mind $B_{y_{n}}(y) \subset B_{2 y_{n}}^{+}\left(y^{\prime}\right)$ ):

$$
f_{B_{y_{n}}(y)}\left|V\left(D u-\hat{\xi} \otimes e_{n}\right)\right|^{2} d x \leq 2^{n-1} 3^{n+2 \sigma} c_{i t}\left[\left(\frac{2 y_{n}}{6 R}\right)^{2 \sigma} \Phi^{2}\left(0,6 R, \xi_{0}(0)\right)+\left(2 y_{n}\right)^{2 \alpha}\right] .
$$

Apart from the explicit estimates for the excess-functions in (58) and (59) in dependency of the radius, we can use our choice in (53) for the radius $R_{0}$, and the smallness condition (54) for the excess function to obtain with $2 y_{n} \leq 4 R_{0}$ according to the choice of $t_{2}$ in (52):

$$
\begin{aligned}
& f_{B_{2 y_{n}}^{+}\left(y^{\prime}\right)}\left|V\left(D u-\hat{\xi} \otimes e_{n}\right)\right|^{2} d x \leq \frac{1}{4} 3^{2 \sigma} c_{i t} t_{2}^{2}, \\
& f_{B_{y_{n}}(y)}\left|V\left(D u-\hat{\xi} \otimes e_{n}\right)\right|^{2} d x \leq 2^{n-3} 3^{2 \sigma} c_{i t} t_{2}^{2} \leq \frac{1}{4} t_{1}^{2} .
\end{aligned}
$$

Since $\left|\hat{\xi} \otimes e_{n}\right| \leq M_{2}=\frac{1}{2} \widehat{M}_{2}$ we only have to make sure that the mean value of $u$ on the ball $B_{y_{n}}(y)$ is bounded by $\frac{1}{2} \widehat{M}_{2}$ for all assumptions in (48) to hold true: the Poincaré inequality in Lemma 3.4, Lemma $3.1,(60)$ and (52) now allows us to estimate (note $t_{2} \leq 1, y_{n} \leq \frac{1}{2}$ ):

$$
\left|(u)_{y, y_{n}}\right| \leq 2^{n} y_{n} f_{B_{2 y_{n}}^{+}\left(y^{\prime}\right)}\left|D u-\hat{\xi} \otimes e_{n}\right| d x+|\hat{\xi}| y_{n} \leq \frac{1}{2} \widehat{M}_{2} .
$$


Via (49) we draw the conclusion: there exists $\Upsilon_{\infty}(y) \in \mathbb{R}^{n N}$ with $\left|\Upsilon_{\infty}(y)\right| \leq \widehat{M}_{2}$, and for all $0<r \leq y_{n}$ we conclude with (59):

$$
\begin{aligned}
f_{B_{r}(y)}\left|V(D u)-V\left(\hat{\xi} \otimes e_{n}\right)\right|^{2} d x & \leq \widehat{c}_{i t}\left[\left(\frac{r}{y_{n}}\right)^{2 \sigma} f_{B_{y_{n}}(y)}\left|V\left(D u-\hat{\xi} \otimes e_{n}\right)\right|^{2} d x+r^{2 \alpha}\right] \\
& \leq \widehat{c}_{i t} c_{i t} 6^{n+2}\left(\left(\frac{r}{6 R}\right)^{2 \sigma} \Phi^{2}\left(0,6 R, \xi_{0}(0)\right)+r^{2 \alpha}\right)
\end{aligned}
$$

Combining the last estimate with (55) and (57) (where we still have to apply Lemma $3.1(\mathrm{v})$ ) we have shown that the assumptions of Theorem 7.1 are satisfied for $V(D u)$. Thus $V(D u) \in C^{0, \alpha}\left(\overline{B_{R}^{+}}, \mathbb{R}^{n N}\right)$, and due to Lemma 3.2 we obtain: $D u \in C^{0, \alpha}\left(\overline{B_{R}^{+}}, \mathbb{R}^{n N}\right)$.

Now the set of regular boundary points can be characterized by a transformation argument:

Proof (of Theorem 2.2): We only want to outline the proof. The transformed function $\tilde{v}=(u-g) \circ$ $\mathcal{T}^{-1} \in W_{\Gamma}^{1, p}\left(B_{r}^{+}, \mathbb{R}^{N}\right)$ (see chapter 2) is a weak solution of $\operatorname{div} \widetilde{A}(\cdot, v, D v)=0$ on $B_{r}^{+}$where the radius $r$ is chosen sufficiently small (in fact we require $B_{1 /(\sqrt{2} \rho)}^{+} \subset \mathcal{T}\left(\Omega \cap B_{\rho}\right) \subset B_{\sqrt{2} \rho}^{+}$for all $\rho \leq \sqrt{2} r$ ). The coefficients $\widetilde{A}$ satisfy the structure conditions analogous to (H1)-(H4). Thus we are in the situation of the last theorem which characterizes the set of regular boundary points in the model situation of a half-ball.

Analogously to the proof of Theorem 7.2 we define $\tilde{\xi}(0, \rho) \in \mathbb{R}^{N}$ by

$$
V(\tilde{\xi}(0, \rho))=\left(V\left(D_{n}(u-g)\right)\right)_{0, \rho}=f_{\Omega \cap B_{\rho}} V\left(D_{n}(u-g)\right) d x .
$$

Using the transformation formula we calculate (cf. [2], proof of Satz 7.5):

$$
\liminf _{\rho \rightarrow 0^{+}} f_{B_{\rho}^{+}}\left|V\left(D u-\tilde{\xi}(0, \sqrt{8} \rho) \otimes e_{n}\right)\right|^{2} d y=0, \quad \limsup _{\rho \rightarrow 0^{+}}|\tilde{\xi}(0, \rho)| \leq c(M)
$$

for some $M \geq 2$. These conditions are comparable to the definition of the sets $\Sigma_{1}^{\prime}$ and $\Sigma_{2}^{\prime}$ from above. Choosing the radius smaller if required, we conclude as in Theorem 7.2 (with $\tilde{v}, \tilde{\xi}(0, \cdot), \min \{\alpha, \beta, \tau\}$ instead of $u, \xi(0, \cdot), \alpha): D \tilde{v}$ is Hölder-continuous with exponent $\min \{\alpha, \beta, \tau\}$ on the half-ball $\overline{B_{R}^{+}}$for some $0<R<1$. Since $\mathcal{T}$ is a transformation of class $C^{1, \tau}$ this gives the desired result.

\section{The inhomogeneous case}

We close by briefly considering the case of inhomogeneous systems of the form

$$
-\operatorname{div} A(\cdot, u, D u)=B(\cdot, u, D u) \quad \text { in } \Omega,
$$

where $B: \bar{\Omega} \times \mathbb{R}^{N} \times \mathbb{R}^{n N} \rightarrow \mathbb{R}^{N}$ is a Carathéodory function, i. e., measurable in $x$ and continuous in $(u, z)$ obeying a controllable or a natural growth condition.

Let us first assume a controllable growth condition, i. e.

$$
\text { (B1) }
$$

$$
|B(x, u, z)| \leq L\left(1+|z|^{2}\right)^{\frac{p-1}{2}}
$$$$
\forall(x, u, z) \in \bar{\Omega} \times \mathbb{R}^{N} \times \mathbb{R}^{n N} .
$$

Transforming the system as indicated at the end of section 2, we can restrict ourselves again to the modelcase $\Omega=B^{+}$of an upper half-ball as far as the boundary regularity is concerned (with $\widetilde{B}$ still satisfying (B1)). In the Caccioppoli inequality in Lemma 5.1 and in Lemma 6.1 of the approximate $\mathcal{A}$-harmonicity an additional integral $f_{B_{\rho}^{+}\left(x_{0}\right)} B(\cdot, u, D u) \cdot \varphi d x$ appears and needs to be estimated:

We chose $\varphi=\eta^{2}\left(u-\xi x_{n}\right)$ as a test function in Lemma 5.1 and get for the new integrand called $I V$

$$
I V \leq \varepsilon|V(D u-X)|^{2} \eta^{2}+L c\left(p, \frac{L}{\varepsilon}, M\right)\left(\left|V\left(\frac{u-\xi x_{n}}{\rho}\right)\right|^{2}+\rho^{2 \alpha}\right)
$$




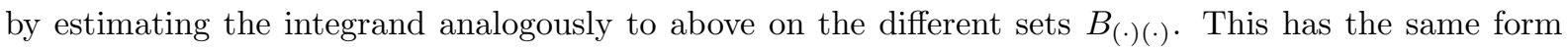
as the estimates $(22)$ and $(23)$ of the terms $I-I I I$. In the second lemma, we consider an arbitrary test function $\varphi \in C_{0}^{\infty}\left(B_{\rho}^{+}, \mathbb{R}^{N}\right)$ with $\sup _{B_{\rho}^{+}}|D \varphi| \leq 1$; this means we may use the fact that $\sup _{B_{\rho}^{+}}|\varphi| \leq \rho$ to obtain

$$
I V \leq L\left(1+|z|^{2}\right)^{\frac{p-1}{2}} \rho \leq L c(M)\left(|V(D u-X)|^{2}+\rho^{\alpha}\right)
$$

as in (27). Hence, Lemma 6.1 is valid in this setting as well.

Instead of (B1) we may require that the inhomogeneity $B$ fulfills a natural growth condition

$$
|B(x, u, z)| \leq L_{1}\left(M_{u}\right)+L_{2}\left(M_{u}\right)|z|^{p} \quad \forall(x, u, z) \in \bar{\Omega} \times \mathbb{R}^{N} \times \mathbb{R}^{n N} \text { with }|u| \leq M_{u} .
$$

In this setting we have to study bounded weak solutions $u \in W^{1, p}\left(\Omega, \mathbb{R}^{N}\right) \cap L^{\infty}\left(\Omega, \mathbb{R}^{N}\right)$. Then the weak formulation corresponding to (3) holds for all functions $\varphi \in W_{0}^{1, p}\left(\Omega, \mathbb{R}^{N}\right) \cap L^{\infty}\left(\Omega, \mathbb{R}^{N}\right)$. We further require $\|u\|_{\infty} \leq M_{u}$ for some $M_{u}>0$ which satisfies $2 L_{2}\left(M_{u}\right) M_{u}<\nu$. In this setting the transformation requires a little more care. For sufficiently small radius $r>0$ we transform the system and obtain: $\tilde{v}=u \circ \mathcal{T}^{-1}(y)-g \circ \mathcal{T}^{-1}(y) \in W_{\Gamma}^{1, p}\left(B_{r}^{+}, \mathbb{R}^{N}\right)$ (as in section 2) is a solution of $\operatorname{div} \widetilde{A}(\cdot, \tilde{v}, D \tilde{v})=\widetilde{B}(\cdot, \tilde{v}, D \tilde{v})$ in $B_{r}^{+}$. Here, the transformed coefficients satisfy structure conditions analogous to (H1),(H2),(H3)* and (H4) where

$$
\text { (H3) }^{*} \quad D_{z} A(x, u, z) \lambda \cdot \lambda \geq \nu\left(\bar{\nu}^{-2}+|z|^{2}\right)^{\frac{p-2}{2}}|\lambda|^{2} \quad \forall \lambda \in \mathbb{R}^{n N}
$$

for $\bar{\nu} \geq 1$ depending on $M_{u}, L_{1}\left(M_{u}\right)$ and $\|D g\|_{\infty}$ (which, of course, implies (H3) for some $\tilde{\nu} \in(0, \nu]$ ). This modified condition is needed due to the fact that we reduce - in contrast to [20] - to zero-boundary-values. Furthermore, there holds $\left|\tilde{v}+g \circ \mathcal{T}^{-1}\right| \leq M_{u}$, and $2 \widetilde{L}_{2}\left(M_{u}\right) M_{u}<\nu$ is still satisfied by the choice of a sufficiently small radius. The latter boundedness condition combined with the new version $(\mathrm{H} 3)^{*}$ of the ellipticity assumption now allows us to estimate the remaining term in the Caccioppoli-inequality, cf. [20], Lemma 4.3, (for the sake of simplicity we write $u, g$ instead of $\tilde{v}, g \circ \mathcal{T}^{-1}$ ): similar to the estimate (20) we deduce the lower bound

$$
\begin{aligned}
f_{B_{\rho}^{+}\left(x_{0}\right)}[A(\cdot, u, D u)-A(\cdot, u, X)] \cdot(D u-X) \eta^{2} d x \\
\quad \geq f_{B_{\rho}^{+}\left(x_{0}\right)} \nu\left(\bar{\nu}^{-2}+\left(1+\delta^{-1}\right)|X|^{2}+(1+\delta)|D u-X|^{2}\right)^{\frac{p-2}{2}}|D u-X|^{2} \eta^{2} d x
\end{aligned}
$$

for some $\delta \in(0,1)$. This time we derive for the integral over $I V$ :

$$
\begin{aligned}
f_{B_{\rho}^{+}\left(x_{0}\right)} I V d x \leq & L_{1}\left(M_{u}\right)(1+\delta)\left(2 M_{u}+\left(\|D g\|_{L^{\infty}}+|\xi|\right) \rho\right) \\
& f_{B_{\rho}^{+}\left(x_{0}\right)}\left(\bar{\nu}^{-2}+\left(1+\delta^{-1}\right)|X|^{2}+(1+\delta)|D u-X|^{2}\right)^{\frac{p-2}{2}}|D u-X|^{2} \eta^{2} d x \\
& +c\left(M, L_{1}\left(M_{u}\right), L_{2}\left(M_{u}\right), \bar{\nu}, \delta^{-1}\right) f_{B_{\rho}^{+}\left(x_{0}\right)}\left(\left|V\left(\frac{u-\xi x_{n}}{\rho}\right)\right|^{2}+\rho^{2 \alpha}\right) d x
\end{aligned}
$$

and, using $2 \widetilde{L}_{2}\left(M_{u}\right) M_{u}<\nu$, absorption of the first integral on the right-hand side is possible provided $\rho$ and $\delta$ are small. Hence, Lemma 5.1 is still valid under the additional boundedness condition, and in the proof of Lemma 6.1 the inhomogeneity can be handled as in the situation of controllable growth. From this point on we may now proceed (apart from the new dependencies of the constants and choosing the radius smaller if necessary) as in the homogeneous situation to obtain:

Theorem 8.1: Let $u \in W^{1, p}\left(\Omega, \mathbb{R}^{N}\right)$ be a weak solution of the inhomogeneous system (61) under the hypotheses (H1)-(H5) and one of the following assumptions on the inhomogeneity

1. $B(\cdot, \cdot, \cdot)$ obeys a controllable growth condition (B1),

2. $B(\cdot, \cdot, \cdot)$ obeys a natural growth condition (B2) and $u \in L^{\infty}\left(\Omega, \mathbb{R}^{N}\right)$ with $\|u\|_{L^{\infty}\left(\Omega, \mathbb{R}^{N}\right)} \leq M_{u}$ such that $2 L_{1}\left(M_{u}\right) M_{u}<\nu$;

then the conclusions of the main theorems 2.1 and 2.2 follow. 


\section{References}

[1] A. Arkhipova, Partial regularity up to the boundary of weak solutions of elliptic systems with nonlinearity q greater than two, J. Math. Sci. (N. Y.) 115 (2003), 2735-2746.

[2] L. Beck, Partielle Regularität für schwache Lösungen nichtlinearer elliptischer Systeme: der subquadratische Fall, diploma thesis, Universität Erlangen-Nürnberg, 2005.

[3] S. Campanato, Elliptic systems with non-linearity q greater or equal to two. Regularity of the solution of the Dirichlet problem, Ann. Math. Pura Appl. Ser. 4147 (1987), 117-150.

[4] C. Capone, L. Greco, and T. Iwaniec, Higher integrability via Riesz transform and interpolation, Nonlinear Anal., Theory Methods Appl., No. 4 (A) 49 (2002), 513-523.

[5] M. Carozza, N. Fusco, and G. Mingione, Partial Regularity of Minimizers of Quasiconvex Integrals with Subquadratic Growth, Ann. Math. Pura Appl. Ser. 4175 (1998), 141-164.

[6] M. Carozza and G. Mingione, Partial regularity of minimizers of quasiconvex integrals with subquadratic growth: the general case, Ann. Pol. Math. 77 (2001), no. 3, 219-243.

[7] F. Colombini, Un teorema di regolarità alla frontiera per soluzioni di sistemi ellittici quasi lineari, Ann. Sc. Norm. Sup. Pisa Ser. III 25 (1971), 15-161.

[8] B. Dacorogna, Direct Methods in the Calculus of Variations, Springer-Verlag, Berlin-Heidelberg-New York, 1989.

[9] E. de Giorgi, Un esempio di estremali discontinue per un problema variazionale di tipo ellittico, Boll. U.M.I. Ser. IV 1 (1968), 135-137.

[10] F. Duzaar and J.F. Grotowski, Optimal interior partial regularity for nonlinear elliptic systems: the method of Aharmonic approximation, Manus. Math. 103 (2000), 267-298.

[11] F. Duzaar, J.F. Grotowski, and M. Kronz, Regularity of almost minimizers of quasi-convex variational integrals with subquadratic growth, Ann. Math. Pura Appl. 11 (2005), no. 4, 421-448.

[12] F. Duzaar, J. Kristensen, and G. Mingione, The existence of regular boundary points for non-linear elliptic systems, J. Reine Angew. Math. 602 (2007), 17-58.

[13] F. Duzaar and G. Mingione, The p-harmonic approximation and the regularity of p-harmonic maps, Calc. Var. 20 (2004), 235-256.

[14] M. Fuchs, Die Green-Matrix für elliptische Systeme zweiter Ordnung in Divergenzform mit stetigen Koeffizienten, Dissertation, Düsseldorf, 1983.

[15] M. Giaquinta, Multiple Integrals in the Calculus of Variations and Nonlinear Elliptic Systems, Princeton University Press, Princeton, New Jersey, 1983.

[16] M. Giaquinta and G. Modica, Almost-everywhere regularity results for solutions of non linear elliptic systems, Manus. Math. 28 (1979), 109-158.

[17] E. Giusti, Direct Methods in the Calculus of Variation, World Scientific Publishing, 2003.

[18] E. Giusti and M. Miranda, Sulla Regolarità delle Soluzioni Deboli di una Classe di Sistemi Ellitici Quasi-lineari, Arch. Rational Mech. Anal. 31 (1968), 173-184.

[19] J.F. Grotowski, Boundary regularity results for nonlinear elliptic systems in divergence form, Habilitationsschrift, Erlangen, 2000.

[20] J.F. Grotowski, Boundary regularity results for nonlinear elliptic systems, Calc. Var. 15 (2002), 353-388.

[21] C. Hamburger, Quasimonotonicity, Regularity and Duality for Nonlinear Systems of Partial Differential Equations, Ann. Math. Pura Appl. 169 (1995), 321-354.

[22] C. Hamburger, Partial boundary regularity of solutions of nonlinear superelliptic systems, Preprint (2004).

[23] P.-A. Ivert, Regularitätsuntersuchungen von Lösungen elliptischer Systeme von quasilinearen Differentialgleichungen zweiter Ordnung, Manus. Math. 30 (1979), 53-88.

[24] G. Mingione, Regularity of minima: an invitation to the Dark Side of the Calculus of Variations, Appl. Math. 51 (2006), no. 4, 355-425.

[25] L. Pepe, Risultati di regolarità parziale per le soluzioni $\mathcal{H}^{1, p} 1<p<2$ di sistemi ellittici quasi lineari, Ann. Univ. Ferrara, N. Ser., Sez. VII 16 (1971), 129-148.

[26] L. Simon, Theorems on Regularity and Singularity of Energy Minimizing Maps, Birkhäuser-Verlag, Basel-BostonBerlin, 1996.

[27] J. Wolf, Regularität schwacher Lösungen nichtlinearer elliptischer und parabolischer Systeme partieller Differentialgleichungen mit Entartung. Der Fall $1<p<2$, Dissertation, Berlin, 2001. 